\title{
Synthesis of the A-B Subunit of Angelmicin B
}

\author{
William T. Lambert ${ }^{\mathrm{a}}$ and William R. Roush*b \\ ${ }^{a}$ Department of Chemistry, University of Michigan, Ann Arbor, MI 48109 and \\ ${ }^{b}$ Department of Medicinal Chemistry, Scripps-Florida, Jupiter, FL 33435 \\ Email: roush@scripps.edu
}

Supporting Information

Part I-Experimental Procedures 
General Methods: All reaction solvents were purified before use. Tetrahydrofuran (THF) and dichloromethane $\left(\mathrm{CH}_{2} \mathrm{Cl}_{2}\right)$ were purified by passing through a solvent column composed of activated A-1 alumina. Chlorotriethylsilane (TESCl), triethylamine $\left(\mathrm{Et}_{3} \mathrm{~N}\right), 2,6$ lutidine, and pyridine (py) were distilled from $\mathrm{CaH}_{2}$ before use. Triisopropylsilyl trifluoromethanesulfonate (TIPSOTf), $t$-butyldimethylsilyl trifluoromethanesulfonate (TBSOTf), oxalyl chloride, and $\square$-benzyloxyacetaldehyde were distilled prior to use. Unless indicated, all other chemicals were used as purchased without further purification.

Physical Properties and Spectroscopic Measurements: Proton nuclear magnetic resonance $\left({ }^{1} \mathrm{H}\right.$ NMR) spectra and carbon-13 $\left({ }^{13} \mathrm{C}\right)$ NMR spectra were recorded on a Varian Inova-500 spectrometer at $500 \mathrm{MHz}$ and $125 \mathrm{MHz}$, respectively, or a Varian Inova-400 spectrometer at $400 \mathrm{MHz}$ and $100 \mathrm{MHz}$, respectively. The proton signal of residual chloroform ( $\square$ 7.26) or tetramethylsilane (TMS; $\square$ 0.00) was used as the internal reference for ${ }^{1} \mathrm{H}$ NMR spectra. Proton signal multiplicities are reported as follows: b (broad), s (singlet), $d$ (doublet), $t$ (triplet), q (quartet). Chemical shifts for ${ }^{13} \mathrm{C} N M R$ spectra are reported relative to the center line of the $\mathrm{CDCl}_{3}$ triplet $(\square 77.00)$. Coupling constants are reported in Hz. Infrared (IR) spectra were recorded as thin films on a Perkin-Elmer Spectrum 1000 FTIR. Mass spectra were recorded on a ZVG 70-250-S spectrometer manufactured by Micromass Corp. (Manchester UK).

Analytical thin layer chromatography (TLC) was performed using Kieselgel $60 \mathrm{~F}_{254}$ glass plates precoated with a $0.25 \mathrm{~mm}$ thickness of silica gel. The TLC plates were visualized with UV light and/or by staining with $p$-anisaldehyde solution ( $p$-anisaldehyde in ethanolic sulfuric acid). Flash column chromatography was performed using Kieselgel 60 (230-400 mesh) silica gel.

HPLC purifications were performed using an HPLC system composed of two Rainin HPXL pumps connected to Dynamax ${ }^{\square}$ axial compression columns packed with Rainin $60 \AA$ irregular silica gel. Samples were loaded into the system with a $2 \mathrm{~mL}$ Rheodyne 7125 injector and were detected using a Rainin Dynamax ${ }^{\square}$ UV-C detector. Integration of the various signals was performed using the reprocessing program within the Dynamax ${ }^{\square}$ HPC Method Manager.

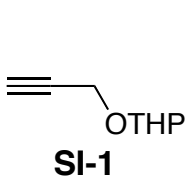

1. $n$-BuLi, THF, $-78^{\circ} \mathrm{C}$;
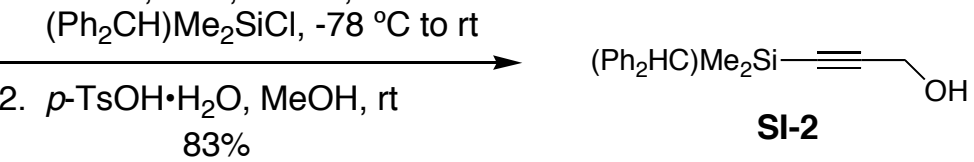

Alcohol SI-2. To a solution of alkyne SI-1 (4.09 g, $29.2 \mathrm{mmol})$ in THF (40 mL) at -78 ${ }^{\circ} \mathrm{C}$ was added $n$-BuLi (12.2 $\mathrm{mL}$ of a $2.4 \mathrm{M}$ solution in hexanes, $29.2 \mathrm{mmol}$ ). The mixture was stirred for $15 \mathrm{~min}$ at $-78^{\circ} \mathrm{C}$, then a solution of benzhydryldimethylchlorosilane ${ }^{1}(7.62 \mathrm{~g}, 29.2$ $\mathrm{mmol})$ in THF $(18 \mathrm{~mL})$ was added via cannula. The reaction mixture was warmed to $0{ }^{\circ} \mathrm{C}$ and maintained at this temperature for $1 \mathrm{~h}$. The mixture was diluted with sat. aq. $\mathrm{NH}_{4} \mathrm{Cl}$, extracted with $\mathrm{Et}_{2} \mathrm{O}$, and the organic layer was washed with brine and dried $\left(\mathrm{MgSO}_{4}\right)$. Concentration of the filtrate in vacuo yielded a yellow oil which was carried forward without further purification. An analytical sample of this intermediate THP ether (SI-1b) for characterization was obtained by

${ }^{1}$ Peng, Z.-H.; Woerpel, K. A. Org. Lett. 2000, $2,1379$. 
flash column chromatography (5\% EtOAc in hexanes): ${ }^{1} \mathbf{H}$ NMR (400 MHz, $\left.\mathrm{CDCl}_{3}\right) \square 0.16(\mathrm{~s}$, $6 \mathrm{H}), 1.44-1.68(\mathrm{~m}, 4 \mathrm{H}), 1.70-1.78(\mathrm{~m}, 1 \mathrm{H}), 1.78-1.90(\mathrm{~m}, 1 \mathrm{H}), 3.49-3.55(\mathrm{~m}, 1 \mathrm{H}), 3.60(\mathrm{~s}, 1 \mathrm{H})$, 3.84 (ddd, $J=12.0,9.0,3.0 \mathrm{~Hz}, 1 \mathrm{H}), 4.28(\mathrm{~s}, 2 \mathrm{H}), 4.78$ (at, $J=3.3 \mathrm{~Hz}, 1 \mathrm{H}), 7.13-7.18(\mathrm{~m}, 2 \mathrm{H})$, 7.24-7.30 (m, 4H), 7.33-7.37 (m, 4H); ${ }^{13} \mathbf{C}$ NMR (100 MHz, $\left.\mathrm{CDCl}_{3}\right) \square-1.7,19.3,25.6,30.5$, 44.9, 54.8, 62.2, 89.4, 96.7, 104.2, 125.7, 128.5, 129.2, 142.1; FTIR (thin film) $\square_{\max } 3024,2944$, 2176, 1598, 1494, 1251, 1120, 1029, 846, 816, $700 \mathrm{~cm}^{-1}$; HRMS (ESI) calcd for $\mathrm{C}_{23} \mathrm{H}_{28} \mathrm{O}_{2} \mathrm{Si}$ $[\mathrm{M}+\mathrm{Na}]^{+} 387.1756 \mathrm{~m} / \mathrm{z}$, found $387.1747 \mathrm{~m} / \mathrm{z}$.

To a solution of the crude THP ether isolated above (SI-1b; $29.2 \mathrm{mmol})$ in $\mathrm{MeOH}$ (58 $\mathrm{mL})$ at $0{ }^{\circ} \mathrm{C}$ was added $p$ - TsOH $\bullet \mathrm{H}_{2} \mathrm{O}(111 \mathrm{mg}, 0.584 \mathrm{mmol})$. The solution was warmed to room temperature and stirred at this temperature overnight. The reaction was quenched by the addition of solid $\mathrm{NaHCO}_{3}(520 \mathrm{mg})$, filtered, and the filtrate was concentrated in vacuo. Purification of the residue by flash column chromatography (5\% to $10 \%$ to $20 \%$ EtOAc in hexanes) yielded alcohol SI-2 (6.8 g, 24 mmol, 83\% over two steps from SI-1) as a light yellow oil: ${ }^{1} \mathbf{H}$ NMR $\left(400 \mathrm{MHz}, \mathrm{CDCl}_{3}\right) \square 0.18(\mathrm{~s}, 6 \mathrm{H}), 1.49(\mathrm{t}, J=6.2 \mathrm{~Hz}, \underline{\mathrm{OH}}), 3.61(\mathrm{~s}, 1 \mathrm{H}), 4.26(\mathrm{~d}, J=6.2 \mathrm{~Hz}$, 2H), 7.14-7.20 (m, 2H), 7.26-7.31 (m, 4H), 7.33-7.36 (m, 4H); ${ }^{13} \mathbf{C}$ NMR $\left(100 \mathrm{MHz}, \mathrm{CDCl}_{3}\right) \square$ 1.8, 44.9, 51.9, 89.3, 106.4, 125.7, 128.6, 129.2, 142.0; FTIR (thin film) $\square_{\max } 3544,3513-3124$ (br), 3024, 2960, 2176, 1597, 1494, 1448, 1251, 1036, 984, 846, 780, $701 \mathrm{~cm}^{-1}$; HRMS (ESI) calcd for $\mathrm{C}_{18} \mathrm{H}_{20} \mathrm{OSi}[\mathrm{M}+\mathrm{Na}]^{+} 303.1181 \mathrm{~m} / \mathrm{z}$, found $303.1171 \mathrm{~m} / \mathrm{z}$.

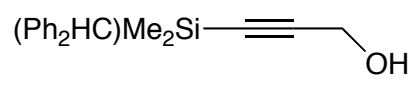

SI-2

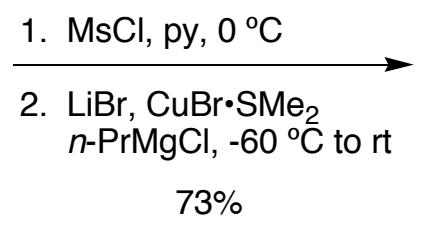

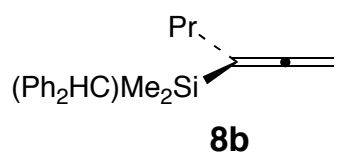

8b

Allene 8b. ${ }^{2}$ To a solution of methanesulfonyl chloride $(3.0 \mathrm{~mL}, 39 \mathrm{mmol})$ in pyridine $(20$ $\mathrm{mL})$ at $0{ }^{\circ} \mathrm{C}$ was added a pre-cooled $\left(0^{\circ} \mathrm{C}\right)$ solution of alcohol SI-2 $(5.5 \mathrm{~g}, 20 \mathrm{mmol})$ in $\mathrm{CH}_{2} \mathrm{Cl}_{2}$ $(20 \mathrm{~mL})$ dropwise via cannula. This mixture was stirred for $1 \mathrm{~h}$ at $0{ }^{\circ} \mathrm{C}$, then was diluted with $\mathrm{H}_{2} \mathrm{O}(100 \mathrm{~mL})$ and extracted with $\mathrm{Et}_{2} \mathrm{O}$. The organic layer was washed with sat. aq. $\mathrm{CuSO}_{4}(2 \square$ $100 \mathrm{~mL})$ to remove pyridine, washed with brine, dried $\left(\mathrm{MgSO}_{4}\right)$, and concentrated in vacuo to afford $5.8 \mathrm{~g}$ (16.2 mmol, 83\% yield) of an orange oil. The crude mesylate prepared in this way was used in the next step without further purification. An analytical sample for characterization was purified by flash column chromatography (20\% to $30 \%$ EtOAc in hexanes) of this residue: ${ }^{1}$ H NMR $\left(500 \mathrm{MHz}, \mathrm{CDCl}_{3}\right) \square 0.19$ (s, 6H), 2.88 (s, 3H), 3.62 (s, 1H), 4.84 (s, 2H), 7.15-7.19 (m, 2H), 7.25-7.32 (m, 8H); ${ }^{13} \mathbf{C}$ NMR (125 MHz, $\left.\mathrm{CDCl}_{3}\right) \square-1.9,39.1,44.5,58.4,94.3,99.2$, 125.9, 128.5, 128.7, 129.1, 129.2, 141.5; FTIR (thin film) $\square_{\max } 3025,2960,2927,2185,1597$, 1493, 1448. 1365, 1252, 1176, 1028, 946, 805, $703 \mathrm{~cm}^{-1}$; HRMS (ESI) calcd for $\mathrm{C}_{19} \mathrm{H}_{22} \mathrm{O}_{3} \mathrm{SSi}$ $[\mathrm{M}+\mathrm{Na}]^{+} 381.0957 \mathrm{~m} / \mathrm{z}$, found $381.0948 \mathrm{~m} / \mathrm{z}$.

\footnotetext{
${ }^{2}$ The procedure for the synthesis of $\mathbf{8 b}$ was adapted from the synthesis of the phenyldimethylsilyl-substituted analogue 8a: Heo, J.-N.; Micalizio, G. C.; Roush, W. R. Org. Lett. 2003, 5, 1693.
} 
To a solution of $\mathrm{LiBr}(1.70 \mathrm{~g}, 19.7 \mathrm{mmol})$ and $\mathrm{CuBr} \cdot \mathrm{SMe}_{2}(4.05 \mathrm{~g}, 19.7 \mathrm{mmol})$ in THF $(25 \mathrm{~mL})$ at $-10{ }^{\circ} \mathrm{C}$ was added $n$ - $\mathrm{PrMgCl}\left(9.9 \mathrm{~mL}\right.$ of a $2 \mathrm{M}$ solution in $\left.\mathrm{Et}_{2} \mathrm{O}, 19.7 \mathrm{mmol}\right)$ dropwise via syringe pump over $1 \mathrm{~h}$. The resulting heterogeneous, dark purple mixture was cooled to -60 ${ }^{\circ} \mathrm{C}$ and a solution of the mesylate from the preceding paragraph $(6.41 \mathrm{~g}, 17.9 \mathrm{mmol})$ in THF (20 $\mathrm{mL}$ ) was added via cannula. The mixture was stirred at $-60^{\circ} \mathrm{C}$ for $30 \mathrm{~min}$, then was warmed to room temperature and stirred for another $30 \mathrm{~min}$. The reaction mixture was then poured into sat. aq. $\mathrm{NH}_{4} \mathrm{Cl}\left(100 \mathrm{~mL}\right.$, adjusted to $\mathrm{pH} \sim 8$ with $\left.\mathrm{NH}_{4} \mathrm{OH}\right)$, and the biphasic mixture was vigorously stirred overnight. The organic layer was then washed with sat. aq. $\mathrm{NH}_{4} \mathrm{Cl}(\mathrm{pH} \sim 8)$ and brine, dried $\left(\mathrm{MgSO}_{4}\right)$, and concentrated in vacuo to afford a reddish oil. Purification of this residue by flash column chromatography (100\% hexanes) yielded allene $\mathbf{8 b}$ (4.79 g, $15.6 \mathrm{mmol}$, 73\% over two steps from alcohol SI-2) as a clear, colorless oil: ${ }^{1} \mathbf{H}$ NMR $\left(500 \mathrm{MHz}, \mathrm{CDCl}_{3}\right) \square 0.11$ (s, $6 \mathrm{H}), 0.81(\mathrm{t}, J=7.4 \mathrm{~Hz}, 3 \mathrm{H}), 1.37$ (sextet, $J=7.4 \mathrm{~Hz}, 2 \mathrm{H}), 1.66-1.71(\mathrm{~m}, 2 \mathrm{H}), 3.66(\mathrm{~s}, 1 \mathrm{H}), 4.28$ $(\mathrm{t}, J=3.5 \mathrm{~Hz}, 2 \mathrm{H}), 7.11-7.16(\mathrm{~m}, 2 \mathrm{H}), 7.22-7.26(\mathrm{~m}, 8 \mathrm{H}) ;{ }^{13} \mathbf{C}$ NMR $\left(100 \mathrm{MHz}, \mathrm{CDCl}_{3}\right) \square-3.3$, 14.0, 22.2, 31.0, 44.9, 69.7, 93.3, 125.4, 127.9, 128.4, 129.1, 129.5, 133.2, 142.6, 209.6; FTIR (thin film) $\square_{\max } 3024,2958,1926,1598,1494,1448,1250,1120,1075,1033,1002,830,811$, $700 \mathrm{~cm}^{-1}$; HRMS (EI) calcd for $\mathrm{C}_{21} \mathrm{H}_{26} \mathrm{Si}[\mathrm{M}]^{+} 306.1804 \mathrm{~m} / \mathrm{z}$, found $306.1801 \mathrm{~m} / \mathrm{z}$.

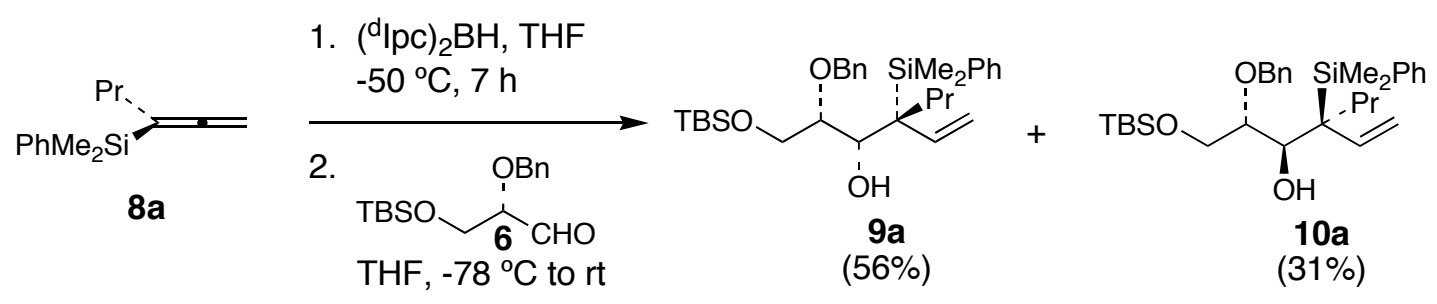

Alcohols 9a and 10a. A flame-dried roundbottom flask was charged with $\left({ }^{\mathrm{d}} \mathrm{Ipc}\right)_{2} \mathrm{BH}^{3}$ (1.32 $\mathrm{g}, 4.63 \mathrm{mmol})$, and THF $(15 \mathrm{~mL})$ was added. The resulting suspension was stirred vigorously for $1 \mathrm{~h}$ at room temperature, at which point the solution became homogeneous. ${ }^{4}$ This solution was cooled to $-50{ }^{\circ} \mathrm{C}$, and allene $8 \mathbf{a}(1.00 \mathrm{~g}, 4.63 \mathrm{mmol})$ in THF (3 mL) was added via cannula. The resulting solution was maintained at $-50{ }^{\circ} \mathrm{C}$ for $7 \mathrm{~h}$ with the aid of an immersion cooler. At this point, the mixture was cooled to $-78{ }^{\circ} \mathrm{C}$, then aldehyde $6(907 \mathrm{mg}, 3.09 \mathrm{mmol})$ in THF ( $3 \mathrm{~mL})$ was added. The mixture was allowed to warm to room temperature overnight. The mixture was concentrated, then EtOAc, $35 \%$ aq. $\mathrm{H}_{2} \mathrm{O}_{2}$, and $\mathrm{pH} 7$ phosphate buffer ( $2 \mathrm{~mL}$ each) were added, and the biphasic mixture was stirred vigorously for $24 \mathrm{~h}$. ${ }^{5}$ The mixture was then diluted with $\mathrm{H}_{2} \mathrm{O}$ and extracted with $\mathrm{Et}_{2} \mathrm{O}$. The organic layer was washed with brine, dried $\left(\mathrm{MgSO}_{4}\right)$, and concentrated in vacuo. The crude product was purified by flash column chromatography (3\% to 5\% EtOAc in hexanes), giving alcohol 9a (492 mg, $0.961 \mathrm{mmol}, 31 \%$ )

\footnotetext{
${ }^{3}$ Prepared from (+)- $\square$-pinene: (a) Brown, H. C.; Singaram, B. J. Org. Chem. 1984, 49, 945. (b) Brown, H. C.; Joshi, N. N. J. Org. Chem. 1988, 53, 4059.

${ }^{4}$ Since $(\mathrm{Ipc})_{2} \mathrm{BH}$ itself is insoluble in most aprotic solvents, it is likely that this compound suffers disproportionation in THF before giving the observed homogeneous mixture. This issue will be examined in future experiments. We thank a referee for helpful suggestions in this regard.

${ }^{5}$ This step was necessary to hydrolyze the intermediate boronate esters; use of more forcing conditions (aq. $\mathrm{NaOH}, \mathrm{H}_{2} \mathrm{O}_{2}$; aq. $\mathrm{NaBO}_{3}$ ) gave significant amounts of Peterson elimination products (e.g., SI-3).
} 
and alcohol 10a (879 mg, $1.72 \mathrm{mmol}, 56 \%)$ as clear oils. Data for 9a: [प] $\mathbf{l}_{\mathbf{D}}{ }^{\mathbf{2 4}}-1.2\left(\mathrm{c} 1.0, \mathrm{CHCl}_{3}\right)$; ${ }^{1}$ H NMR $\left(500 \mathrm{MHz}, \mathrm{CDCl}_{3}\right) \square-0.04(\mathrm{~s}, 3 \mathrm{H}),-0.02(\mathrm{~s}, 3 \mathrm{H}), 0.33(\mathrm{~s}, 3 \mathrm{H}), 0.44(\mathrm{~s}, 3 \mathrm{H}), 0.83$ (s, $9 \mathrm{H}), 0.87(\mathrm{t}, J=7.5 \mathrm{~Hz}, 3 \mathrm{H}), 1.42-1.55(\mathrm{~m}, 3 \mathrm{H}), 1.72-1.80(\mathrm{~m}, 1 \mathrm{H}), 2.70(\mathrm{~d}, J=8.5 \mathrm{~Hz}, \mathrm{OH})$, 3.58 (as, 3H), $3.75(\mathrm{~d}, J=8.5 \mathrm{~Hz}, 1 \mathrm{H}), 4.38(\mathrm{~d}, J=11.0 \mathrm{~Hz}, 1 \mathrm{H}), 4.64(\mathrm{~d}, J=11.0 \mathrm{~Hz}, 1 \mathrm{H}), 4.82$ $(\mathrm{dd}, J=18.0,1.5 \mathrm{~Hz}, 1 \mathrm{H}), 5.04(\mathrm{dd}, J=11.5,1.5 \mathrm{~Hz}, 1 \mathrm{H}), 5.75(\mathrm{dd}, J=18.0,11.5 \mathrm{~Hz}, 1 \mathrm{H})$, 7.25-7.35 (m, 8H), 7.59-7.63 (m, 2H); ${ }^{13} \mathbf{C}$ NMR (100 MHz, $\left.\mathrm{CDCl}_{3}\right) \square-5.6,-2.4,-2.3,15.5,18.2$, $19.5,25.9,32.6,42.3,63.0,72.0,72.6,77.9,112.3,127.2,127.7,127.9,128.3,128.6,135.2$, 138.3, 138.4, 141.2; FTIR (thin film) $\square_{\max } 3560,3070,2957,2929,2858,1618,1471,1428$, $1402,1253,1109,837,775,701 \mathrm{~cm}^{-1}$; HRMS (ESI) calcd for $\mathrm{C}_{30} \mathrm{H}_{48} \mathrm{O}_{3} \mathrm{Si}_{2}[\mathrm{M}+\mathrm{Na}]^{+} 535.3040$ $\mathrm{m} / \mathrm{z}$, found $535.3052 \mathrm{~m} / \mathrm{z}$.

Data for 10a: [प] ${ }_{\mathbf{D}}{ }^{24}-3.0\left(\right.$ c 2.1, $\left.\mathrm{CHCl}_{3}\right) ;{ }^{1} \mathbf{H}$ NMR $\left(500 \mathrm{MHz}, \mathrm{CDCl}_{3}\right) \square-0.00(\mathrm{~s}, 3 \mathrm{H})$, $0.02(\mathrm{~s}, 3 \mathrm{H}), 0.33(\mathrm{~s}, 3 \mathrm{H}), 0.43(\mathrm{~s}, 3 \mathrm{H}), 0.87(\mathrm{~s}, 9 \mathrm{H}), 0.89(\mathrm{t}, J=7.5 \mathrm{~Hz}, 3 \mathrm{H}), 3.17(\mathrm{~d}, J=4.0 \mathrm{~Hz}$, $\mathrm{OH}), 3.44(\mathrm{dt}, J=5.5,3.5 \mathrm{~Hz}, 1 \mathrm{H}), 3.76\left(\underline{\mathrm{ABX}}, J_{\mathrm{AB}}=11.5 \mathrm{~Hz}, J_{\mathrm{AX}}=4.0 \mathrm{~Hz}, 1 \mathrm{H}\right), 3.80(\mathrm{ABX}$, $\left.J_{\mathrm{AB}}=11.5 \mathrm{~Hz}, J_{\mathrm{BX}}=4.0 \mathrm{~Hz}, 1 \mathrm{H}\right), 3.92(\mathrm{dd}, J=5.5,4.5 \mathrm{~Hz}, 1 \mathrm{H}), 4.34(\underline{\mathrm{AB}}, J=11.0 \mathrm{~Hz}, 1 \mathrm{H})$, $4.38(\mathrm{AB}, J=11.0 \mathrm{~Hz}, 1 \mathrm{H}), 4.76(\mathrm{dd}, J=17.5,1.5 \mathrm{~Hz}, 1 \mathrm{H}), 4.93(\mathrm{dd}, J=11.0,1.5 \mathrm{~Hz}, 1 \mathrm{H}), 5.81$ $(\mathrm{dd}, J=17.5,11.0 \mathrm{~Hz}, 1 \mathrm{H}), 7.19-7.35(\mathrm{~m}, 8 \mathrm{H}), 7.58-7.61(\mathrm{~m}, 2 \mathrm{H}) ;{ }^{13} \mathbf{C}$ NMR $\left(100 \mathrm{MHz}, \mathrm{CDCl}_{3}\right)$ $\square-5.6,-5.5,-2.6,-2.3,15.5,18.1,19.2,25.8,32.0,41.8,64.0,71.2,77.9,79.7,110.4,127.3$, 127.4, 127.8, 128.2 128.6, 135.0, 138.5, 138.6, 140.9; FTIR (thin film) $\square_{\max } 3480,3069,2956$, 2930, 2857, 1620, 1471, 1428, 1254, 1087, 836, 776, 736, $701 \mathrm{~cm}^{-1}$; HRMS (ESI) calcd for $\mathrm{C}_{30} \mathrm{H}_{48} \mathrm{O}_{3} \mathrm{Si}_{2}[\mathrm{M}+\mathrm{Na}]^{+} 535.3040 \mathrm{~m} / \mathrm{z}$, found $535.3041 \mathrm{~m} / \mathrm{z}$.

The syn stereochemistry of $9 \mathbf{a}$ and $\mathbf{1 0 a}$ was proven by conversion of each to the same (E)-diene (SI-3) via base-induced Peterson elimination:

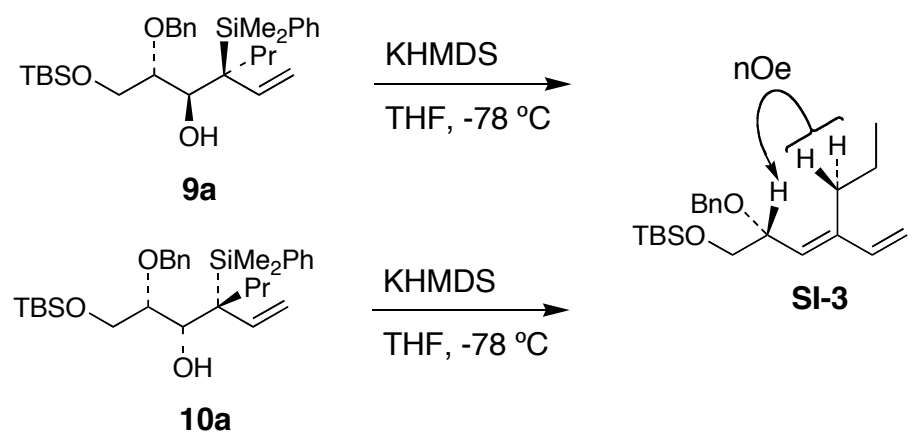

The carbinol stereochemistry of 9a and 10a was secured by Mosher ester analysis ${ }^{6}$ of their oxidative desilylation ${ }^{7}$ derivatives SI-4 and SI-5, respectively:

\footnotetext{
${ }^{6}$ Ohtani, I.; Kusumi, T.; Kashman, Y.; Kakisawa, H. J. Am. Chem. Soc. 1991, 113, 4092.

${ }^{7}$ Roush, W. R.; Grover, P. T. Tetrahedron 1992, 48, 1981.
} 

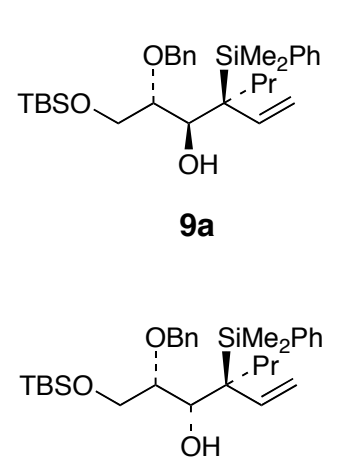

$10 a$
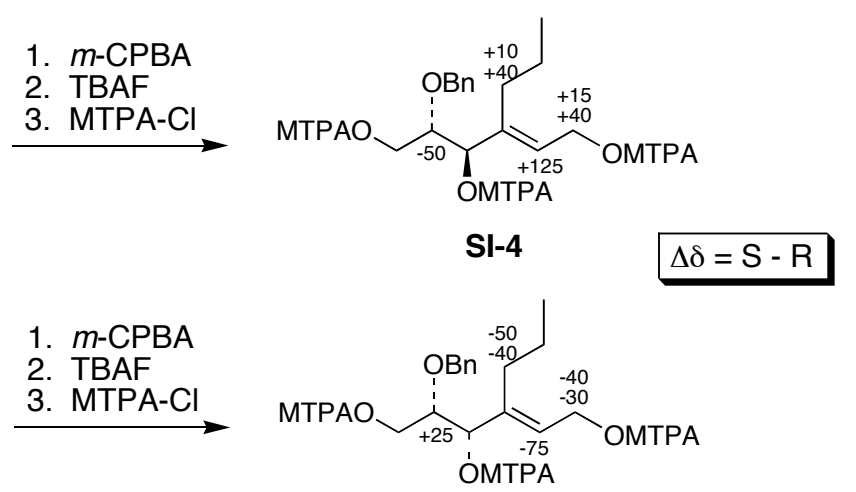

SI-5
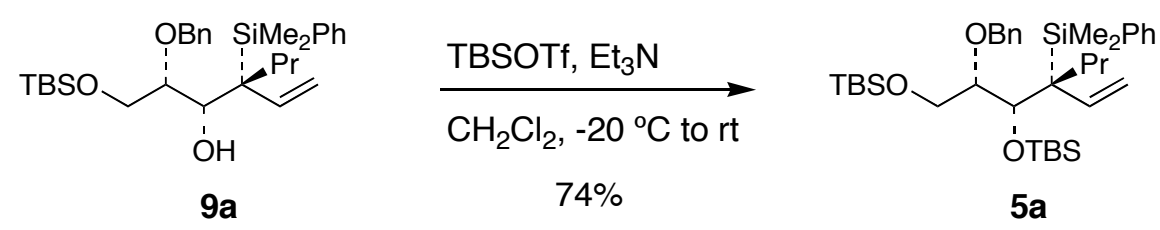

Allylsilane 5a. To a solution of $\mathrm{Et}_{3} \mathrm{~N}(0.844 \mathrm{~mL}, 6.10 \mathrm{mmol})$ in $\mathrm{CH}_{2} \mathrm{Cl}_{2}(1.5 \mathrm{~mL})$ at room temperature was added TBSOTf $(0.70 \mathrm{~mL}, 3.1 \mathrm{mmol})$. The solution was stirred for $30 \mathrm{~min}$ at room temperature, cooled to $-20^{\circ} \mathrm{C}$, and a solution of alcohol 9a $(745 \mathrm{mg}, 1.46 \mathrm{mmol})$ in $\mathrm{CH}_{2} \mathrm{Cl}_{2}(1.5 \mathrm{~mL})$ was added via cannula. The reaction mixture was then warmed to room temperature and stirred at this temperature overnight before it was diluted with sat. aq. $\mathrm{NaHCO}_{3}$. The mixture was then extracted with $\mathrm{Et}_{2} \mathrm{O}$ and the organic layer was washed with brine, dried $\left(\mathrm{MgSO}_{4}\right)$, and concentrated in vacuo. Purification of the residue by flash column chromatography (1\% EtOAc in hexanes) afforded 5a $(672 \mathrm{mg}, 1.07 \mathrm{mmol}, 73 \%)$ as a clear, colorless oil. Data for 5a: $[\square]_{\mathbf{D}}{ }^{24}+7.7\left(c 1.6, \mathrm{CHCl}_{3}\right) ;{ }^{1} \mathbf{H} \mathbf{~ N M R}\left(500 \mathrm{MHz}, \mathrm{CDCl}_{3}\right) \square-0.05(\mathrm{~s}$, $3 \mathrm{H}),-0.02(\mathrm{~s}, 3 \mathrm{H}), 0.07(\mathrm{~s}, 3 \mathrm{H}), 0.08(\mathrm{~s}, 3 \mathrm{H}), 0.31(\mathrm{~s}, 3 \mathrm{H}), 0.47(\mathrm{~s}, 3 \mathrm{H}), 0.83(\mathrm{~s}, 9 \mathrm{H}), 0.87(\mathrm{t}, J=$ $7.5 \mathrm{~Hz}, 3 \mathrm{H}), 0.93(\mathrm{~s}, 9 \mathrm{H}), 1.36-1.50(\mathrm{~m}, 3 \mathrm{H}), 1.66-1.74(\mathrm{~m}, 1 \mathrm{H}), 3.63\left(\underline{\mathrm{ABX}}, J_{\mathrm{AB}}=10.0 \mathrm{~Hz}, J_{\mathrm{AX}}\right.$ $=7.0 \mathrm{~Hz}, 1 \mathrm{H}), 3.67\left(\mathrm{ABX}, J_{\mathrm{AB}}=10.0 \mathrm{~Hz}, J_{\mathrm{BX}}=5.5 \mathrm{~Hz}, 1 \mathrm{H}\right), 3.74(\mathrm{ddd}, J=7.0,6.0,1.0 \mathrm{~Hz}$, $1 \mathrm{H}), 3.85(\mathrm{~d}, J=11.5 \mathrm{~Hz}, 1 \mathrm{H}), 4.21(\mathrm{~d}, J=0.5 \mathrm{~Hz}, 1 \mathrm{H}), 4.34(\mathrm{~d}, J=11.5 \mathrm{~Hz}, 1 \mathrm{H}), 4.78(\mathrm{dd}, J=$ $17.5,1.5 \mathrm{~Hz}, 1 \mathrm{H}), 4.97(\mathrm{dd}, J=11.0,1.5 \mathrm{~Hz}, 1 \mathrm{H}), 6.26(\mathrm{dd}, J=17.5,11.0 \mathrm{~Hz}, 1 \mathrm{H}), 7.14-7.18$ (m, 2H), 7.19-7.38 (m, 6H), 7.54-7.57 (m, 2H); ${ }^{13}$ C NMR (125 MHz, $\left.\mathrm{CDCl}_{3}\right) \square-5.37,-5.36$, 3.5, -3.2, -2.8, -1.9, 14.9, 18.27, 18.29, 19.1, 26.0, 26.3, 34.0, 42.7, 63.0, 71.0, 72.7, 81.7, 111.4, 127.0, 127.2, 127.4, 127.9, 128.7, 135.5, 139.1, 139.3, 143.2; FTIR (thin film) $\square_{\max } 3070,2957$, 2930, 2857, 1618, 1472, 1428, 1361, 1253, 1085, 1026, 835, 774, 736, $701 \mathrm{~cm}^{-1}$; HRMS (ESI) calcd for $\mathrm{C}_{36} \mathrm{H}_{62} \mathrm{O}_{3} \mathrm{Si}_{3}[\mathrm{M}+\mathrm{Na}]^{+} 649.3905 \mathrm{~m} / \mathrm{z}$, found $649.3910 \mathrm{~m} / \mathrm{z}$.

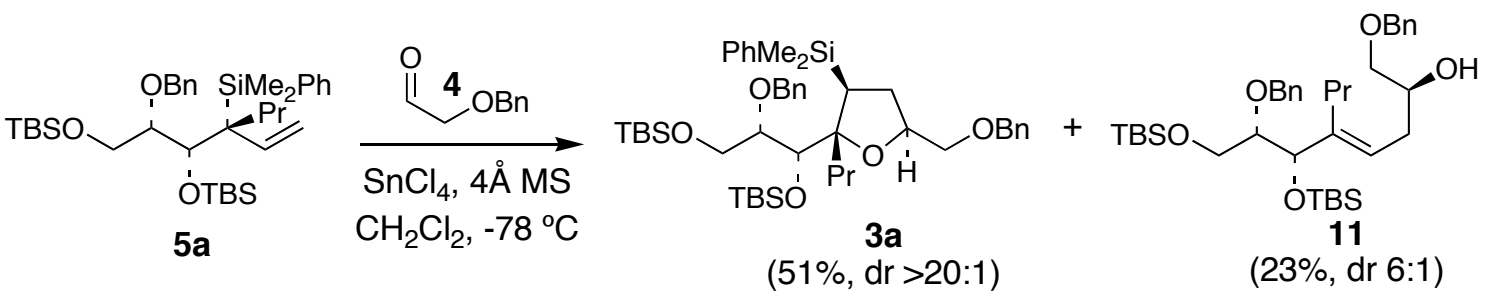


Tetrahydrofuran 3a and Homoallylic Alcohol 11. To a solution of allylsilane 5a (323 $\mathrm{mg}, 0.516 \mathrm{mmol})$ in $\mathrm{CH}_{2} \mathrm{Cl}_{2}(1.0 \mathrm{~mL})$ at room temperature was added $4 \AA \mathrm{MS}(52 \mathrm{mg})$ and $\square-$ benzyloxyacetaldehyde $(4,155 \mathrm{mg}, 0.147 \mathrm{~mL}, 1.03 \mathrm{mmol})$. The mixture was stirred at room temperature for $10 \mathrm{~min}$, then was cooled to $-78{ }^{\circ} \mathrm{C}$ and $\mathrm{SnCl}_{4}(67 \mathrm{mg}, 0.030 \mathrm{~mL}, 0.26 \mathrm{mmol})$ was added dropwise via syringe. The mixture was stirred at this temperature for $1.5 \mathrm{~h}$ before $\mathrm{Et}_{3} \mathrm{~N}$ $(0.365 \mathrm{mg}, 0.500 \mathrm{~mL}, 3.61 \mathrm{mmol})$ was added; the mixture was then allowed to warm to room temperature. The resulting slurry was diluted with sat. aq. $\mathrm{NaHCO}_{3}$, stirred at room temperature for $20 \mathrm{~min}$, and extracted with $\mathrm{Et}_{2} \mathrm{O}$. The combined organic layers were washed with brine, dried $\left(\mathrm{MgSO}_{4}\right)$, and concentrated in vacuo. Purification of the residue by flash chromatography ( $1 \%$ to $3 \%$ to $10 \%$ EtOAc in hexanes) afforded tetrahydrofuranyl silane 3a (205 mg, 0.264 mmol, 51\%, ca. 90\% pure) and alcohol 11 (75 $\mathrm{mg}, 0.12 \mathrm{mmol}, 23 \%$ ) as a ca. 6:1 mixture of diastereomers (major shown). An analytical sample of $\mathbf{3 a}$ was purified by HPLC (2\% EtOAc in hexanes, $21 \mathrm{~mm}$ Dynamax 60A column, $10 \mathrm{~mL} / \mathrm{min})$. An analytical sample of diastereomerically pure 11 was obtained by HPLC (10\% EtOAc in hexanes, $21 \mathrm{~mm}$ Dynamax 60A column, $10 \mathrm{~mL} / \mathrm{min})$. Data for 3a: []$_{\mathbf{D}}{ }^{24}-10\left(c 2.9, \mathrm{CHCl}_{3}\right) ;{ }^{1} \mathbf{H} \mathbf{~ N M R}\left(500 \mathrm{MHz}, \mathrm{CDCl}_{3}\right)$ $\square-0.13(\mathrm{~s}, 3 \mathrm{H}),-0.11(\mathrm{~s}, 3 \mathrm{H}), 0.02(\mathrm{~s}, 3 \mathrm{H}), 0.04(\mathrm{~s}, 3 \mathrm{H}), 0.27(\mathrm{~s}, 3 \mathrm{H}), 0.31(\mathrm{~s}, 3 \mathrm{H}), 0.72(\mathrm{t}, J=7.5$ $\mathrm{Hz}, 3 \mathrm{H}), 0.85$ (s, 9H), $0.89(\mathrm{~s}, 9 \mathrm{H}), 1.08-1.24(\mathrm{~m}, 2 \mathrm{H}), 1.40-1.54(\mathrm{~m}, 2 \mathrm{H}), 1.67$ (dt, J = 12.0, 4.0 $\mathrm{Hz}, 1 \mathrm{H}), 1.89$ (quint, $J=6.0 \mathrm{~Hz}, 1 \mathrm{H}), 2.53(\mathrm{dd}, J=13.5,7.5 \mathrm{~Hz}, 1 \mathrm{H}), 3.43$ (dd, $J=10.0,5.0 \mathrm{~Hz}$, $1 \mathrm{H}), 3.51(\mathrm{dd}, J=10.0,6.0 \mathrm{~Hz}, 1 \mathrm{H}), 3.64(\mathrm{ddd}, J=7.5,5.0,1.5 \mathrm{~Hz}, 1 \mathrm{H}), 3.69(\mathrm{~d}, J=5.0 \mathrm{~Hz}$, $1 \mathrm{H}), 3.95(\mathrm{dd}, J=11.5,7.5 \mathrm{~Hz}, 1 \mathrm{H}), 4.08-4.15(\mathrm{~m}, 2 \mathrm{H}), 4.54(\underline{\mathrm{AB}}, J=12.0 \mathrm{~Hz}, 1 \mathrm{H}), 4.57(\mathrm{AB}, J$ $=12.0 \mathrm{~Hz}, 1 \mathrm{H}), 4.64(\mathrm{~d}, J=12.0 \mathrm{~Hz}, 1 \mathrm{H}), 4.81(\mathrm{~d}, J=12.0 \mathrm{~Hz}, 1 \mathrm{H}), 7.23-7.29(\mathrm{~m}, 4 \mathrm{H}), 7.29-$ 7.37 (m, 9H), 7.37-7.40 (m, 2H); ${ }^{13} \mathbf{C}$ NMR (125 MHz, $\left.\mathrm{CDCl}_{3}\right) \square-5.40,-5.38,-5.0,-4.5,-2.2$, 2.1, 14.6, 18.1, 18.2, 18.3, 25.95, 26.03, 32.6, 33.9, 41.4, 65.4, 73.0, 73.1, 73.4, 77.2, 78.5, 85.8, $90.8,127.2,127.3,127.56,127.60,127.8,128.17,128.22,128.9,134.1,138.67,138.69,139.5$; FTIR (thin film) $\square_{\max } 3068,3030,2955,2929,2857,1472,1361,1256,1079,864,836,774$, 735, $698 \mathrm{~cm}^{-1}$; HRMS (ESI) calcd for $\mathrm{C}_{45} \mathrm{H}_{72} \mathrm{O}_{5} \mathrm{Si}_{3}[\mathrm{M}+\mathrm{Na}]^{+} 799.4585 \mathrm{~m} / z$, found 799.4581 $\mathrm{m} / \mathrm{z}$.

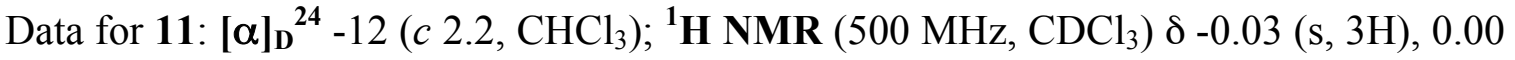
$(\mathrm{s}, 3 \mathrm{H}), 0.00(\mathrm{~s}, 3 \mathrm{H}), 0.02(\mathrm{~s}, 3 \mathrm{H}), 0.88(\mathrm{~s}, 18 \mathrm{H}), 0.90(\mathrm{t}, J=7.0 \mathrm{~Hz}, 3 \mathrm{H}), 1.33-1.46(\mathrm{~m}, 2 \mathrm{H})$, 1.95-2.08 (m, 2H), 2.21-2.32 (m, 2H), $2.32(\mathrm{~d}, J=4.0 \mathrm{~Hz}, \mathrm{OH}), 3.36(\mathrm{dd}, J=9.5,7.5 \mathrm{~Hz}, 1 \mathrm{H})$, 3.43 (ddd, $J=8.5,5.0,4.0 \mathrm{~Hz}, 1 \mathrm{H}), 3.49-3.54(\mathrm{~m}, 2 \mathrm{H}), 3.71$ (dd, $J=11.0,4.0 \mathrm{~Hz}, 1 \mathrm{H}), 3.83$ (qt, $J=7.0,3.5 \mathrm{~Hz}, 1 \mathrm{H}), 4.18(\mathrm{~d}, J=5.0 \mathrm{~Hz}, 1 \mathrm{H}), 4.52(\mathrm{as}, 2 \mathrm{H}), 4.65(\mathrm{AB}, J=12.0 \mathrm{~Hz}, 1 \mathrm{H}), 4.69$ $(\mathrm{AB}, J=12.0 \mathrm{~Hz}, 1 \mathrm{H}), 5.49(\mathrm{t}, J=7.5 \mathrm{~Hz}, 1 \mathrm{H}), 7.22-7.27(\mathrm{~m}, 1 \mathrm{H}), 7.27-7.37(\mathrm{~m}, 9 \mathrm{H}) ;{ }^{13} \mathbf{C}$ NMR $\left(125 \mathrm{MHz}, \mathrm{CDCl}_{3}\right) \square-5.4,-5.3,-5.0,-4.6,14.6,18.18,18.19,19.2,22.7,25.87,25.90,30.4,31.7$, $63.5,70.5,73.4,74.0,75.2,82.6,122.1,127.2,127.6,127.69,127.72,128.1,128.4,138.0,139.2$, 141.7; FTIR (thin film) $\square_{\max } 3468$ (br), 3065, 3031, 2956, 2929, 2857, 1472, 1462, 1454, 1361, 1253, 1094, 836, 776, 734, $697 \mathrm{~cm}^{-1}$; HRMS (ESI) calcd for $\mathrm{C}_{37} \mathrm{H}_{62} \mathrm{O}_{5} \mathrm{Si}_{2}[\mathrm{M}+\mathrm{Na}]^{+} 665.4034$ $\mathrm{m} / \mathrm{z}$, found $665.4030 \mathrm{~m} / z$.

Selected ${ }^{1} \mathrm{H}$ nOe enhancements for silane $\mathbf{3 a}$ : 


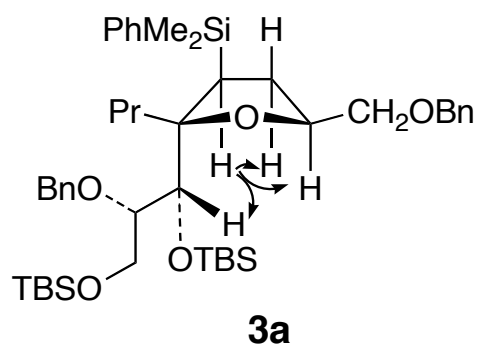

The double bond geometry and carbinol stereochemistry of $\mathbf{1 1}$ were determined by ${ }^{1} \mathrm{H}$ nOe analysis and Mosher ester analysis, respectively, as shown:
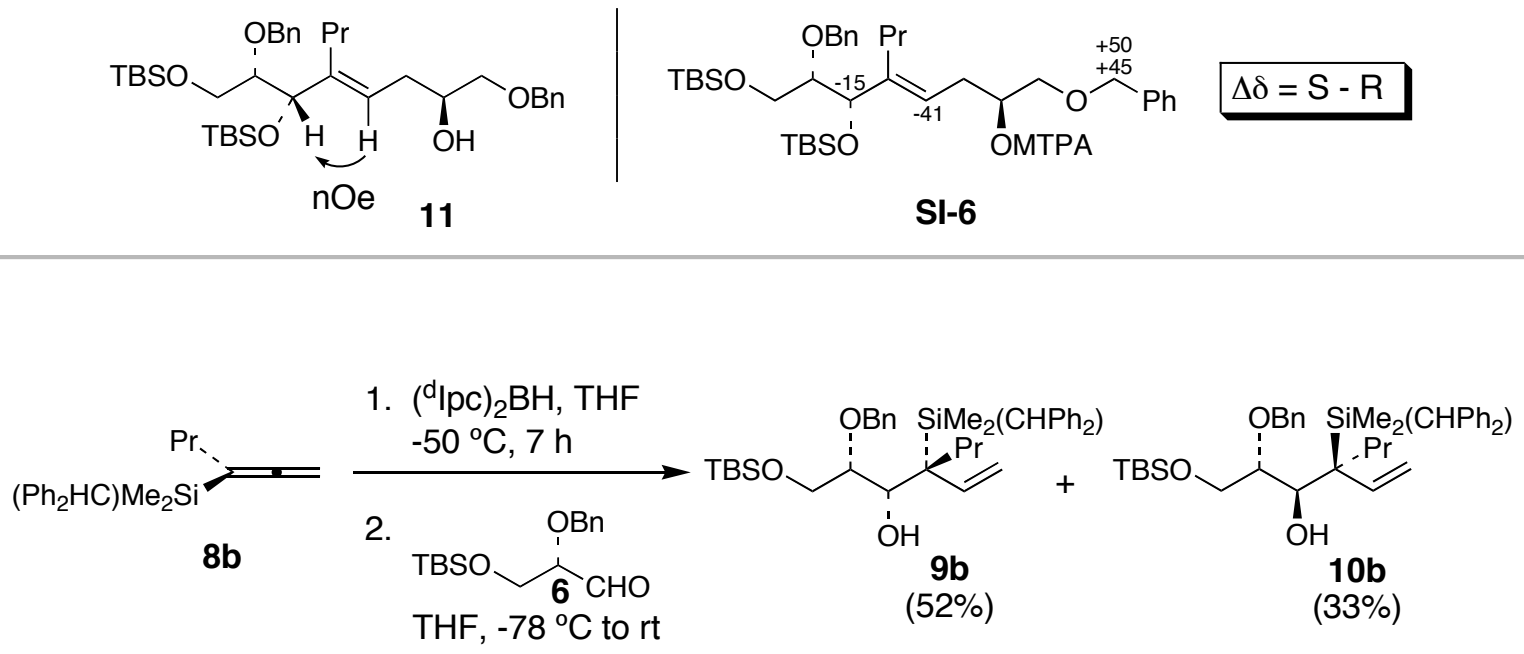

Alcohols 9b and 10b. A flame-dried roundbottom flask was charged with $\left({ }^{\mathrm{d}} \mathrm{Ipc}\right)_{2} \mathrm{BH}^{3}$ ( $858 \mathrm{mg}, 3.00 \mathrm{mmol})$, and THF $(8 \mathrm{~mL})$ was added. The resulting suspension was stirred vigorously for $1 \mathrm{~h}$ at room temperature, at which point the solution became homogeneous. ${ }^{4}$ This solution was cooled to $-50{ }^{\circ} \mathrm{C}$, and allene $\mathbf{8 b}(918 \mathrm{mg}, 3.00 \mathrm{mmol})$ in THF $(2 \mathrm{~mL})$ was added via cannula. The resulting solution was maintained at $-50{ }^{\circ} \mathrm{C}$ for $7 \mathrm{~h}$ with the aid of an immersion cooler. At this point, the mixture was cooled to $-78{ }^{\circ} \mathrm{C}$ before aldehyde $6(588 \mathrm{mg}, 2.00 \mathrm{mmol})$ in THF $(3 \mathrm{~mL})$ was added. The mixture was allowed to warm to room temperature overnight. The mixture was then concentrated, EtOAc, $35 \%$ aq. $\mathrm{H}_{2} \mathrm{O}_{2}$, and $\mathrm{pH} 7$ phosphate buffer $(1.5 \mathrm{~mL}$ each) were added, and the biphasic mixture was stirred vigorously for $24 \mathrm{~h}^{3}$ The mixture was then diluted with $\mathrm{H}_{2} \mathrm{O}$ and extracted with $\mathrm{Et}_{2} \mathrm{O}$. The organic layer was washed with brine, dried $\left(\mathrm{MgSO}_{4}\right)$, and concentrated in vacuo to afford a colorless oil. Purification of the crude product by flash chromatography (3\% to 5\% EtOAc in hexanes) yielded alcohol 9b (625 mg, $1.03 \mathrm{mmol}$, $52 \%)$ and alcohol $\mathbf{1 0 b}(394 \mathrm{mg}, 0.654 \mathrm{mmol}, 33 \%)$ as clear oils. Data for 9b: [D] $\mathbf{b}^{24}+6.2(c 2.5$, $\left.\mathrm{CHCl}_{3}\right) ;{ }^{1} \mathbf{H}$ NMR $\left(500 \mathrm{MHz}, \mathrm{CDCl}_{3}\right) \square 0.04(\mathrm{~s}, 3 \mathrm{H}), 0.04(\mathrm{~s}, 3 \mathrm{H}), 0.11(\mathrm{~s}, 3 \mathrm{H}), 0.15$ (s, 3H), $0.64(\mathrm{t}, J=7.5 \mathrm{~Hz}, 3 \mathrm{H}), 0.89(\mathrm{~s}, 9 \mathrm{H}), 1.11-1.23(\mathrm{~m}, 1 \mathrm{H}), 1.25-1.38(\mathrm{~m}, 2 \mathrm{H}), 1.54(\mathrm{td}, J=14.0$, $5.5 \mathrm{~Hz}, 1 \mathrm{H}), 2.87(\mathrm{~d}, J=3.0 \mathrm{~Hz}, \mathrm{OH}), 3.51(\mathrm{td}, J=6.0,2.0 \mathrm{~Hz}, 1 \mathrm{H}), 3.57\left(\underline{\mathrm{ABX}}, J_{\mathrm{AB}}=10.0 \mathrm{~Hz}\right.$, $\left.J_{\mathrm{AX}}=5.9 \mathrm{~Hz}, 1 \mathrm{H}\right), 3.61\left(\mathrm{ABX}, J_{\mathrm{AB}}=10.0 \mathrm{~Hz}, J_{\mathrm{BX}}=5.1 \mathrm{~Hz}, 1 \mathrm{H}\right), 3.83(\mathrm{dd}, J=8.0,2.0 \mathrm{~Hz}, 1 \mathrm{H})$, $3.94(\mathrm{~s}, 1 \mathrm{H}), 4.48(\mathrm{~d}, J=11.0 \mathrm{~Hz}, 1 \mathrm{H}), 4.71(\mathrm{dd}, J=18.0,1.0 \mathrm{~Hz}, 1 \mathrm{H}), 4.76(\mathrm{~d}, J=11.0 \mathrm{~Hz}, 1 \mathrm{H})$, $4.92(\mathrm{dd}, J=11.0,1.0 \mathrm{~Hz}, 1 \mathrm{H}), 5.52$ (dd, $J=18.0,11.0 \mathrm{~Hz}, 1 \mathrm{H}), 7.06-7.13(\mathrm{~m}, 2 \mathrm{H}), 7.18-7.24$ (m, 4H), 7.27-7.37 (m, 9H); ${ }^{13} \mathbf{C}$ NMR (100 MHz, $\left.\mathrm{CDCl}_{3}\right) \square-5.4,-2.44,-2.35,15.3,18.2,19.5$, 
25.9, 32.6, 43.4, 44.5, 64.2, 72.2, 73.2, 77.6, 112.3, 124.9, 125.2, 127.7, 127.8, 128.1, 128.2, 128.3, 129.1, 129.5, 138.3, 141.2, 143.7, 143.8; FTIR (thin film) $\square_{\max } 3553,3085,3025,2957$, 2857, 1619, 1596, 1494, 1471, 1405, 1251, 1078, 905, 838, 778, 747, $705 \mathrm{~cm}^{-1}$; HRMS (ESI) calcd for $\mathrm{C}_{37} \mathrm{H}_{54} \mathrm{O}_{3} \mathrm{Si}_{2}[\mathrm{M}+\mathrm{Na}]^{+} 625.3509 \mathrm{~m} / \mathrm{z}$, found $625.3509 \mathrm{~m} / \mathrm{z}$.

Data for 10b: [D] ${ }^{24}-16\left(c 1.5, \mathrm{CHCl}_{3}\right) ;{ }^{1} \mathbf{H}$ NMR $\left(500 \mathrm{MHz}, \mathrm{CDCl}_{3}\right) \square 0.05(\mathrm{~s}, 3 \mathrm{H})$, $0.06(\mathrm{~s}, 3 \mathrm{H}), 0.13(\mathrm{~s}, 3 \mathrm{H}), 0.13(\mathrm{~s}, 3 \mathrm{H}), 0.73(\mathrm{t}, J=7.5 \mathrm{~Hz}, 3 \mathrm{H}), 0.91(\mathrm{~s}, 9 \mathrm{H}), 1.23-1.41(\mathrm{~m}, 2 \mathrm{H})$, $1.42-1.57(\mathrm{~m}, 2 \mathrm{H}), 3.33(\mathrm{~d}, J=4.5 \mathrm{~Hz}, \mathrm{OH}), 3.39(\mathrm{aq}, J=5.0 \mathrm{~Hz}, 1 \mathrm{H}), 3.78(\mathrm{dd}, J=11.0,4.0$ $\mathrm{Hz}, 1 \mathrm{H}), 3.84$ (dd, $J=11.0,4.5 \mathrm{~Hz}, 1 \mathrm{H}), 3.92$ (s, 1H), 4.10 (at, $J=4.5 \mathrm{~Hz}, 1 \mathrm{H}), 4.31$ ( $\underline{\mathrm{AB}}, J=$ $11.5 \mathrm{~Hz}, 1 \mathrm{H}), 4.36(\mathrm{AB}, J=11.5 \mathrm{~Hz}, 1 \mathrm{H}), 4.69(\mathrm{dd}, J=18.0,1.5 \mathrm{~Hz}, 1 \mathrm{H}), 4.84(\mathrm{dd}, J=11.0,1.5$ $\mathrm{Hz}, 1 \mathrm{H}), 5.62(\mathrm{dd}, J=18.0,11.0 \mathrm{~Hz}, 1 \mathrm{H}), 7.06-7.13(\mathrm{~m}, 2 \mathrm{H}), 7.18-7.24(\mathrm{~m}, 4 \mathrm{H}), 7.26-7.37(\mathrm{~m}$, 9H); ${ }^{13} \mathbf{C}$ NMR (125 MHz, $\left.\mathrm{CDCl}_{3}\right) \square-5.5,-5.4,-2.6,-2.4,15.4,18.2,19.2,25.8,32.0,42.9,44.1$, $64.0,70.9,78.4,79.2,110.7,125.0,125.1,127.6,128.10,128.12,128.2,129.1,129.5,138.6$, 140.9, 143.78, 143.84; FTIR (thin film) $\square \max 3469,3025,2956,2929,2857,1596,1494,1448$, 1253, 1077, 837, 779, $704 \mathrm{~cm}^{-1}$; HRMS (ESI) calcd for $\mathrm{C}_{37} \mathrm{H}_{54} \mathrm{O}_{3} \mathrm{Si}_{2}[\mathrm{M}+\mathrm{Na}]^{+} 625.3509 \mathrm{~m} / z$, found $625.3516 \mathrm{~m} / \mathrm{z}$.

Stereochemical assignments for $\mathbf{9 b}$ and $\mathbf{1 0 b}$ are based on comparison with the ${ }^{1} \mathrm{H}$ NMR spectra for $9 \mathbf{a}$ and 10a.

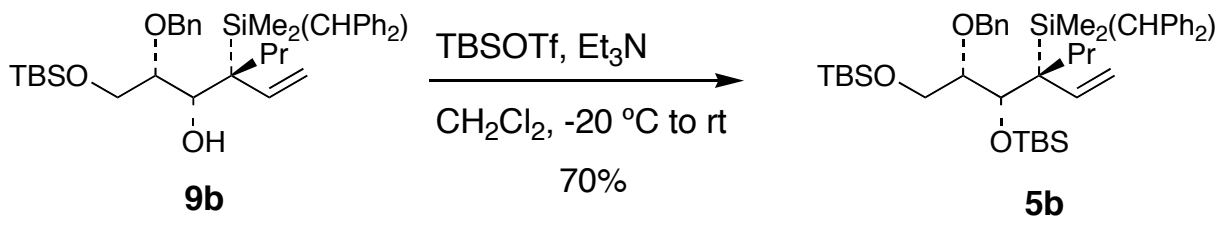

Allylsilane 5b. To a solution of $\mathrm{Et}_{3} \mathrm{~N}(1.10 \mathrm{~mL}, 7.92 \mathrm{mmol})$ in $\mathrm{CH}_{2} \mathrm{Cl}_{2}(2.0 \mathrm{~mL})$ at room temperature was added TBSOTf $(1.36 \mathrm{~mL}, 5.94 \mathrm{mmol})$. The solution was stirred for $30 \mathrm{~min}$ at room temperature, then cooled to $-20^{\circ} \mathrm{C}$ and a solution of alcohol $9 \mathrm{~b}(1.19 \mathrm{~g}, 1.98 \mathrm{mmol})$ in $\mathrm{CH}_{2} \mathrm{Cl}_{2}(2.0 \mathrm{~mL})$ was added via cannula. The reaction mixture was then warmed to room temperature and stirred overnight before sat. aq. $\mathrm{NaHCO}_{3}$ was added. The mixture was then extracted with $\mathrm{Et}_{2} \mathrm{O}$ and the organic layer was washed with brine, dried $\left(\mathrm{MgSO}_{4}\right)$, and concentrated in vacuo. Purification of the residue by flash chromatography (1\% EtOAc in hexanes) afforded $\mathbf{5 b}(990 \mathrm{mg}, 1.38 \mathrm{mmol}, 70 \%)$ as a clear, colorless oil: [D $\mathbf{l}_{\mathbf{D}}{ }^{24}+13(c$ 2.1, $\left.\mathrm{CHCl}_{3}\right) ;{ }^{1} \mathbf{H}$ NMR $\left(500 \mathrm{MHz}, \mathrm{CDCl}_{3}\right) \square-0.01(\mathrm{~s}, 3 \mathrm{H}),-0.00(\mathrm{~s}, 3 \mathrm{H}), 0.06(\mathrm{~s}, 3 \mathrm{H}), 0.07$ (s, 3H), $0.08(\mathrm{~s}, 3 \mathrm{H}), 0.25(\mathrm{~s}, 3 \mathrm{H}), 0.64(\mathrm{t}, J=7.0 \mathrm{~Hz}, 3 \mathrm{H}), 0.85(\mathrm{~s}, 9 \mathrm{H}), 0.91(\mathrm{~s}, 9 \mathrm{H}), 1.11-1.22(\mathrm{~m}, 1 \mathrm{H})$, $1.25-1.45(\mathrm{~m}, 2 \mathrm{H}), 1.49-1.57(\mathrm{~m}, 1 \mathrm{H}), 3.68(\mathrm{dd}, J=9.0,6.0 \mathrm{~Hz}, 1 \mathrm{H}), 3.71(\mathrm{td}, J=6.0,2.0 \mathrm{~Hz}$, $1 \mathrm{H}), 3.75(\mathrm{~s}, 1 \mathrm{H}), 3.80(\mathrm{dd}, J=9.0,5.5 \mathrm{~Hz}, 1 \mathrm{H}), 4.10(\mathrm{~d}, J=1.5 \mathrm{~Hz}, 1 \mathrm{H}), 4.57(\mathrm{~d}, J=11.5 \mathrm{~Hz}$, $1 \mathrm{H}), 4.62(\mathrm{dd}, J=17.5,1.5 \mathrm{~Hz}, 1 \mathrm{H}), 4.75(\mathrm{~d}, J=11.5 \mathrm{~Hz}, 1 \mathrm{H}), 4.83(\mathrm{dd}, J=11.5,1.5 \mathrm{~Hz}, 1 \mathrm{H})$, $6.05(\mathrm{dd}, J=17.5,11.5 \mathrm{~Hz}, 1 \mathrm{H}), 7.04-7.12(\mathrm{~m}, 2 \mathrm{H}), 7.15-7.25(\mathrm{~m}, 5 \mathrm{H}), 7.26-7.37(\mathrm{~m}, 8 \mathrm{H}) ;{ }^{13} \mathbf{C}$ NMR $\left(125 \mathrm{MHz}, \mathrm{CDCl}_{3}\right) \square-5.4,-5.3,-3.3,-3.1,-1.7,-1.3,14.7,18.2,18.3,19.1,26.0,26.4$, $33.4,43.2,43.8,63.6,71.7,74.0,81.3,112.0,124.9,125.1,127.2,127.7,128.07,128.11,128.12$, 129.3, 129.6, 138.9, 142.0, 144.0; FTIR (thin film) $\square_{\max } 3085,3062,3025,2956,2929,2857$, 1596, 1472, 1361, 1253, 1077, 835, 775, $704 \mathrm{~cm}^{-1}$; HRMS (ESI) calcd for $\mathrm{C}_{43} \mathrm{H}_{68} \mathrm{O}_{3} \mathrm{Si}_{3}[\mathrm{M}+$ $\mathrm{Na}]^{+} 739.4374 \mathrm{~m} / \mathrm{z}$, found $739.4360 \mathrm{~m} / \mathrm{z}$. 


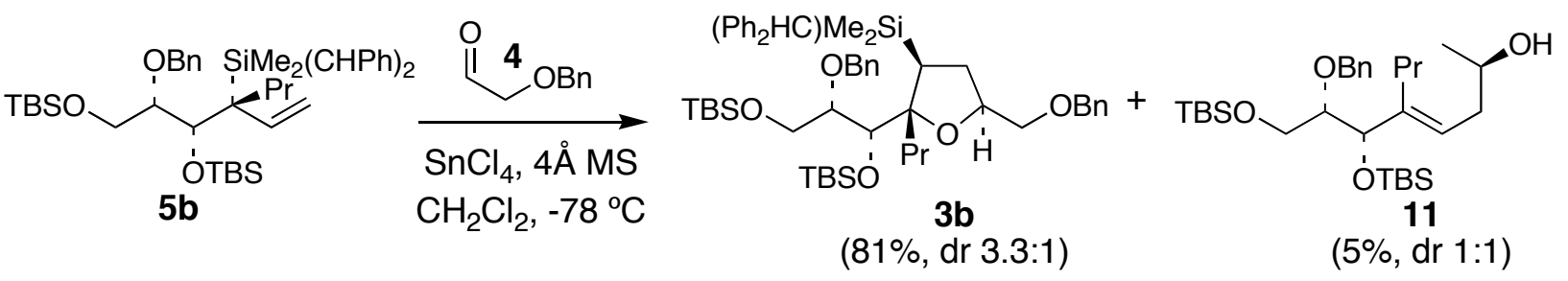

Tetrahydrofuran 3b and Homoallylic Alcohol 11. To a solution of allylsilane 5b (1.51 g, $2.11 \mathrm{mmol})$ in $\mathrm{CH}_{2} \mathrm{Cl}_{2}(4.2 \mathrm{~mL})$ at room temperature were added $4 \AA \mathrm{MS}(210 \mathrm{mg})$ and $\square$ benzyloxyacetaldehyde $(4,633 \mathrm{mg}, 0.603 \mathrm{~mL}, 4.22 \mathrm{mmol})$. The mixture was stirred at room temperature for $10 \mathrm{~min}$, then was cooled to $-78^{\circ} \mathrm{C}$ and $\mathrm{SnCl}_{4}(0.123 \mathrm{~mL}, 1.05 \mathrm{mmol})$ was added dropwise via syringe. The mixture was stirred at $-78^{\circ} \mathrm{C}$ for $1.5 \mathrm{~h}$ before $\mathrm{Et}_{3} \mathrm{~N}(1.0 \mathrm{~g}, 1.4 \mathrm{~mL}, 10$ mmol) was added. The mixture was allowed to warm to room temperature, then was diluted with sat. aq. $\mathrm{NaHCO}_{3}$, stirred at room temperature for 20 min, and extracted with $\mathrm{Et}_{2} \mathrm{O}$. The combined organic layers were washed with brine, dried $\left(\mathrm{MgSO}_{4}\right)$, and concentrated in vacuo. Purification of the residue by flash chromatography (1\% to $3 \%$ to $10 \%$ EtOAc in hexanes) afforded tetrahydrofuranyl silane $\mathbf{3 b}(1.48 \mathrm{~g}, 1.71 \mathrm{mmol}, 81 \%)$ as a $3.3: 1$ mixture of diastereomers ( ${ }^{1} \mathrm{H}$ NMR analysis) and alcohol $11(72 \mathrm{mg}, 0.112 \mathrm{mmol}, 5 \%)$ as a ca. $1: 1$ mixture of diastereomers. An analytical sample of diastereomerically pure $\mathbf{3 b}$ was obtained by HPLC purification (2\% EtOAc in hexanes, $21 \mathrm{~mm}$ Dynamax $60 \mathrm{~A}$ column, $10 \mathrm{~mL} / \mathrm{min})$. Data for $\mathbf{3 b}$ :

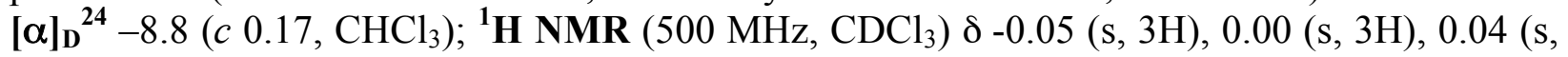
$3 \mathrm{H}), 0.06(\mathrm{~s}, 3 \mathrm{H}), 0.12(\mathrm{~s}, 3 \mathrm{H}), 0.14(\mathrm{~s}, 3 \mathrm{H}), 0.76(\mathrm{t}, J=7.5 \mathrm{~Hz}, 3 \mathrm{H}), 0.87$ (s, 9H), 0.90 (s, 9H), $1.02-1.37(\mathrm{~m}, 4 \mathrm{H}), 1.44-1.55(\mathrm{~m}, 1 \mathrm{H}), 1.62(\mathrm{td}, J=13.5,4.0 \mathrm{~Hz}, 1 \mathrm{H}), 2.24$ (dd, $J=13.5,6.5 \mathrm{~Hz}$, 1H). $3.35(\mathrm{dd}, J=10.0,4.5 \mathrm{~Hz}, 1 \mathrm{H}), 3.36(\mathrm{~s}, 1 \mathrm{H}), 3.41$ (dd, $J=10.0,6.5 \mathrm{~Hz}, 1 \mathrm{H}), 3.65$ (ddd, $J=$ 6.5, 4.0, $1.5 \mathrm{~Hz}, 1 \mathrm{H}), 3.84(\mathrm{~d}, J=4.5 \mathrm{~Hz}, 1 \mathrm{H}), 3.89-3.96(\mathrm{~m}, 1 \mathrm{H}), 3.93(\mathrm{dd}, J=11.5,7.0 \mathrm{~Hz}$, $1 \mathrm{H}), 4.09(\mathrm{dd}, J=11.5,1.5 \mathrm{~Hz}, 1 \mathrm{H}), 4.51(\mathrm{AB}, J=12.0 \mathrm{~Hz}, 1 \mathrm{H}), 4.55(\mathrm{AB}, J=12.0 \mathrm{~Hz}, 1 \mathrm{H})$, $4.67(\mathrm{~d}, J=12.0 \mathrm{~Hz}, 1 \mathrm{H}), 4.77(\mathrm{~d}, J=12.0 \mathrm{~Hz}, 1 \mathrm{H}), 7.05-7.14(\mathrm{~m}, 4 \mathrm{H}), 7.15-7.23(\mathrm{~m}, 6 \mathrm{H}), 7.25-$ $7.41(\mathrm{~m}, 10 \mathrm{H}) ;{ }^{13} \mathbf{C}$ NMR $\left(125 \mathrm{MHz}, \mathrm{CDCl}_{3}\right) \mathrm{\square}-5.4,-4.7,-4.3,-3.2,-1.7,14.7,18.1,18.19$, $18.21,25.9,26.1,32.2,32.3,41.5,46.4,65.3,67.0,72.8,73.1,77.6,78.3,84.9,90.8,125.16$, $125.21,127.3,127.38,127.44,128.0,128.2,128.26,128.28,128.7,128.9,138.8,139.4,142.7$, 142.9; FTIR (thin film) $\square_{\max } 3026,2955,2928,2856,1597,1495,1453,1252,1077,836,775$, 734, $698 \mathrm{~cm}^{-1}$; HRMS (ESI) calcd for $\mathrm{C}_{52} \mathrm{H}_{78} \mathrm{O}_{5} \mathrm{Si}_{3}[\mathrm{M}+\mathrm{Na}]^{+} 889.5055 \mathrm{~m} / z$, found 889.5066 $\mathrm{m} / \mathrm{z}$.

Selected ${ }^{1} \mathrm{H}$ nOe enhancements for tetrahydrofuran $\mathbf{3 b}$ : 


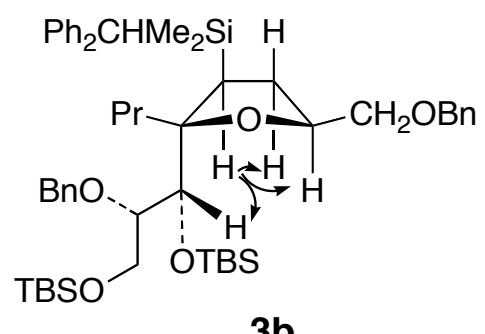

$3 b$

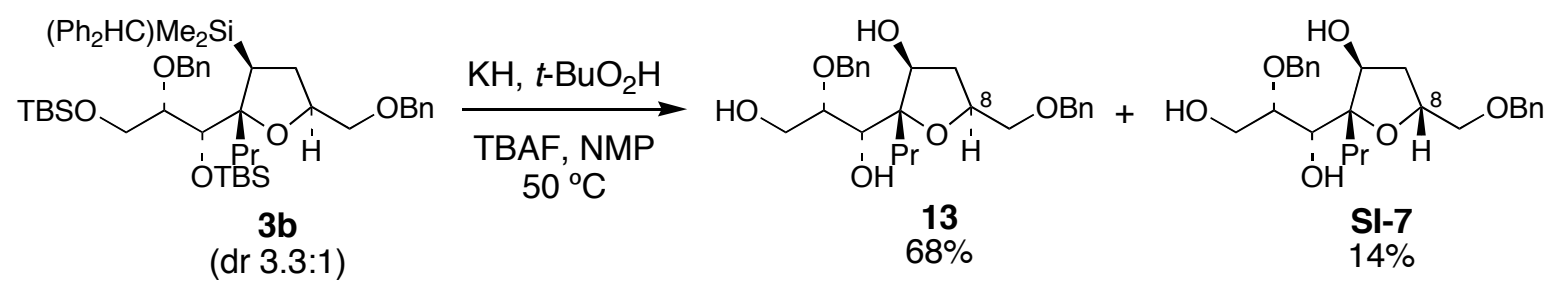

Triols 13 and SI-7. To a solution of tetrahydrofuranyl silane 3b $(896 \mathrm{mg}, 1.03 \mathrm{mmol}$, a $3.3: 1$ mixture of diastereomers) in $N$-methylpyrrolidinone $(5.2 \mathrm{~mL})$ was added $\mathrm{KH}(206 \mathrm{mg}$, $5.15 \mathrm{mmol})$ in one portion followed by $t-\mathrm{BuOOH}(1.03 \mathrm{~mL}$ of a ca. $5 \mathrm{M}$ soln. in decane, ca. 5.15 $\mathrm{mmol}$ ) dropwise via syringe. This mixture was stirred for $15 \mathrm{~min}$ at room temperature, then TBAF $(5.15 \mathrm{~mL}$ of a $1 \mathrm{M}$ soln in THF, $5.15 \mathrm{mmol})$ was added dropwise and the mixture was stirred at room temperature for $1 \mathrm{~h}$ before being placed in a $50{ }^{\circ} \mathrm{C}$ oil bath. The mixture was stirrred at $50{ }^{\circ} \mathrm{C}$ overnight, then was cooled to room temperature, diluted with $\mathrm{H}_{2} \mathrm{O}$, and thoroughly extracted with $\mathrm{Et}_{2} \mathrm{O}$. The combined organic layers were washed with sat. aq. $\mathrm{Na}_{2} \mathrm{~S}_{2} \mathrm{O}_{3}$ and brine, dried $\left(\mathrm{MgSO}_{4}\right)$, and concentrated in vacuo. Purification of the residue by flash chromatography ( $70 \%$ to $85 \%$ EtOAc in hexanes to 100\% EtOAc) afforded triol 13 (301 $\mathrm{mg}, 0.700 \mathrm{mmol}, 68 \%$ ) along with its C8 epimer SI-7 (64 mg, $0.149 \mathrm{mmol}, 14 \%$ ) as colorless

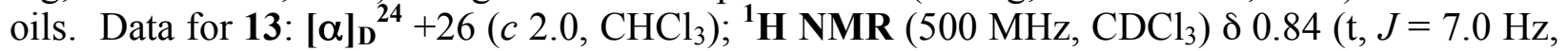
$3 \mathrm{H}), 1.18-1.29(\mathrm{~m}, 1 \mathrm{H}), 1.45-1.54(\mathrm{~m}, 3 \mathrm{H}), 1.79(\mathrm{dt}, J=12.5,8.5 \mathrm{~Hz}, 1 \mathrm{H}), 2.28(\mathrm{dt}, J=14.0$, $13.0 \mathrm{~Hz}, 1 \mathrm{H}$ ), 2.74 (br s, OH), 3.18 (d, $J=4.5 \mathrm{~Hz}, \mathrm{OH}), 3.29$, (br s, OH), 3.38 (dd, $J=10.0,6.0$ $\mathrm{Hz}, 1 \mathrm{H}), 3.50(\mathrm{dd}, J=10.0,2.5 \mathrm{~Hz}, 1 \mathrm{H}), 3.61-3.65(\mathrm{~m}, 1 \mathrm{H}), 3.84(\mathrm{~d}, J=4.0 \mathrm{~Hz}, 1 \mathrm{H}), 3.88$ (dd, $J$ $=12.5,2.5 \mathrm{~Hz}, 1 \mathrm{H}), 4.01(\mathrm{dd}, J=12.5,5.0 \mathrm{~Hz}, 1 \mathrm{H}), 4.15-4.22(\mathrm{~m}, 1 \mathrm{H}), 4.45(\mathrm{~d}, J=10.5 \mathrm{~Hz}$, $1 \mathrm{H}), 4.56(\mathrm{AB}, J=12.0 \mathrm{~Hz}, 1 \mathrm{H}), 4.58(\mathrm{AB}, J=12.5 \mathrm{~Hz}, 1 \mathrm{H}), 4.52-4.62(\mathrm{~m}, 1 \mathrm{H}), 4.84(\mathrm{~d}, J=$ $11.0 \mathrm{~Hz}, 1 \mathrm{H}), 7.26-7.31(\mathrm{~m}, 1 \mathrm{H}), 7.31-7.40(\mathrm{~m}, 9 \mathrm{H}) ;{ }^{13} \mathbf{C}$ NMR $\left(125 \mathrm{MHz}, \mathrm{CDCl}_{3}\right) \mathrm{C} 15.0,16.6$, $33.9,35.1,61.9,71.2,71.3,73.2,73.4,75.8,76.4,77.1,87.8,127.8,128.4,128.5,128.8,136.6$, 137.7; FTIR (thin film) $\square \max 3438$ (br), 3064, 3031, 2958, 2928, 2871, 1497, 1454, 1123, 1067, 738, $699 \mathrm{~cm}^{-1}$; HRMS (ESI) calcd for $\mathrm{C}_{25} \mathrm{H}_{34} \mathrm{O}_{6}[\mathrm{M}+\mathrm{Na}]^{+} 453.2253 \mathrm{~m} / \mathrm{z}$, found $453.2260 \mathrm{~m} / \mathrm{z}$.

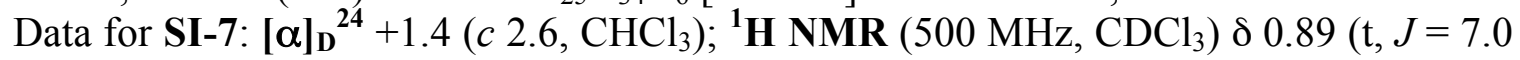
$\mathrm{Hz}, 3 \mathrm{H}), 1.15-1.34(\mathrm{~m}, 2 \mathrm{H}), 1.48-1.60(\mathrm{~m}, 2 \mathrm{H}), 2.08-2.18(\mathrm{~m}, 2 \mathrm{H}), 2.86(\mathrm{~d}, J=2.5 \mathrm{~Hz}, \mathrm{O} \underline{\mathrm{H}})$, 3.25 (dd, $J=10.0,2.5 \mathrm{~Hz}, 1 \mathrm{H}), 3.53-3.56(\mathrm{~m}, 2 \mathrm{H}), 3.57$ (dd, $J=10.0,3.0 \mathrm{~Hz}, 1 \mathrm{H}), 3.93(\mathrm{dt}, J=$ $12.5,2.5 \mathrm{~Hz}, 1 \mathrm{H}), 4.00(\mathrm{~s}, \mathrm{OH}), 4.01(\mathrm{~d}, J=3.0 \mathrm{~Hz}, 1 \mathrm{H}), 4.11-4.19(\mathrm{~m}, 2 \mathrm{H}), 4.39$ (d, $J=10.5$ $\mathrm{Hz}, 1 \mathrm{H}), 4.48(\mathrm{~d}, J=12.5 \mathrm{~Hz}, 1 \mathrm{H}), 4.61(\mathrm{~d}, J=12.5 \mathrm{~Hz}, 1 \mathrm{H}), 4.75(\mathrm{td}, J=9.5,2.5 \mathrm{~Hz}, 1 \mathrm{H}), 4.88$ $(\mathrm{d}, J=11.0 \mathrm{~Hz}, 1 \mathrm{H}), 7.27-7.32(\mathrm{~m}, 3 \mathrm{H}), 7.32-7.37(\mathrm{~m}, 3 \mathrm{H}), 7.37-7.40(\mathrm{~m}, 4 \mathrm{H}) ;{ }^{13} \mathbf{C}$ NMR $(100$ $\left.\mathrm{MHz}, \mathrm{CDCl}_{3}\right) \square 15.0,16.4,33.6,35.6,61.7,69.6,70.7,72.9,73.3,74.4,76.1,78.3,88.7,127.95$, 127.97, 128.5, 128.6, 128.8, 128.9, 136.5, 137.2; FTIR (thin film) $\square_{\max } 3436$ (br), 2957, 2867, 
1455, 1062, 738, $698 \mathrm{~cm}^{-1}$; HRMS (ESI) calcd for $\mathrm{C}_{25} \mathrm{H}_{34} \mathrm{O}_{6}[\mathrm{M}+\mathrm{Na}]^{+} 453.2253 \mathrm{~m} / z$, found $453.2256 \mathrm{~m} / \mathrm{z}$.

The stereochemistry of SI-7 (the C8 epimer of $\mathbf{1 3}$ ) was determined by ${ }^{1} \mathrm{H}$ nOe analysis as shown below:

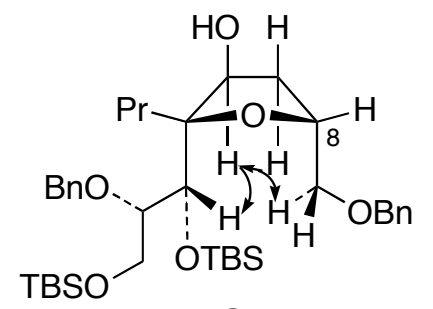

SI-7

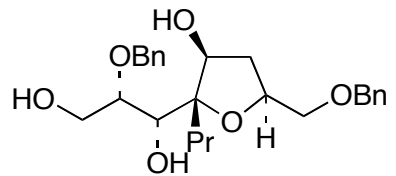

13

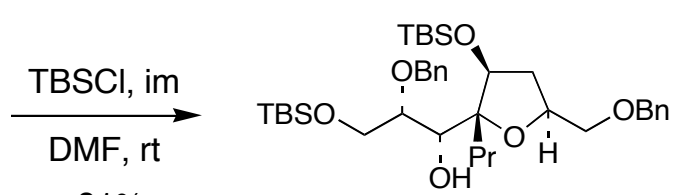

14

Alcohol 14. To a solution of triol $13(726 \mathrm{mg}, 1.69 \mathrm{mmol})$ in DMF (4.2 $\mathrm{mL})$ at room temperature was added imidazole $(1.38 \mathrm{~g}, 20.3 \mathrm{mmol})$ followed by TBSCl $(1.53 \mathrm{mg}, 10.1$ mmol). The reaction mixture was stirred at this temperature for $24 \mathrm{~h}$, at which point it was diluted with $\mathrm{H}_{2} \mathrm{O}$. The mixture was extracted with $\mathrm{Et}_{2} \mathrm{O}$, and the combined organic layers were washed with brine, dried $\left(\mathrm{MgSO}_{4}\right)$, and concentrated in vacuo. Purification of the residue by flash chromatography (5\% to 10\% EtOAc in hexanes) afforded the bis(TBS ether) 14 (1.01 g, $1.53 \mathrm{mmol}, 91 \%)$ as a colorless oil. Data for 14: [0] $]_{\mathbf{D}}{ }^{24}+28\left(\mathrm{c} \mathrm{2.6}, \mathrm{CHCl}_{3}\right) ;{ }^{1} \mathrm{H}$ NMR $(500$ $\left.\mathrm{MHz} \mathrm{CDCl}_{3}\right) \square 0.05(\mathrm{~s}, 3 \mathrm{H}), 0.05(\mathrm{~s}, 3 \mathrm{H}), 0.06(\mathrm{~s}, 6 \mathrm{H}), 0.84(\mathrm{t}, J=7.0 \mathrm{~Hz}, 3 \mathrm{H}), 0.87(\mathrm{~s}, 9 \mathrm{H})$, $0.90(\mathrm{~s}, 9 \mathrm{H}), 1.37-1.48(\mathrm{~m}, 1 \mathrm{H}), 1.50-1.62(\mathrm{~m}, 2 \mathrm{H}), 1.61-1.71(\mathrm{~m}, 2 \mathrm{H}), 2.29(\mathrm{dt}, J=12.5,7.0 \mathrm{~Hz}$, $1 \mathrm{H}), 2.67$ (d, $J=7.5 \mathrm{~Hz}, \mathrm{OH}), 3.41(\mathrm{dd}, J=7.5,2.5 \mathrm{~Hz}, 1 \mathrm{H}), 3.45$ (dd, $J=10.0,5.0 \mathrm{~Hz}, 1 \mathrm{H})$, $\left.3.60(\mathrm{dd}, J=10.0,6.5 \mathrm{~Hz}, 1 \mathrm{H}), 3.73\left(\underline{\mathrm{ABX}}, J_{\mathrm{AB}}=10.0 \mathrm{~Hz}, 1 \mathrm{H}\right), J_{\mathrm{AX}}=6.5 \mathrm{~Hz}, 1 \mathrm{H}\right), 3.74(\mathrm{ABX}$, $\left.J_{\mathrm{AB}}=10.0 \mathrm{~Hz}, J_{\mathrm{BX}}=5.5 \mathrm{~Hz}, 1 \mathrm{H}\right), 3.82(\mathrm{td}, J=6.0,2.5 \mathrm{~Hz}, 1 \mathrm{H}), 4.11(\mathrm{qd}, J=6.5,5.5 \mathrm{~Hz}, 1 \mathrm{H})$, $4.49(\mathrm{dd}, J=6.0,5.0 \mathrm{~Hz}, 1 \mathrm{H}), 4.54-4.60(\mathrm{~m}, 2 \mathrm{H}), 4.63(\mathrm{~d}, J=11.0 \mathrm{~Hz}, 1 \mathrm{H}), 4.83(\mathrm{~d}, J=11.0 \mathrm{~Hz}$,

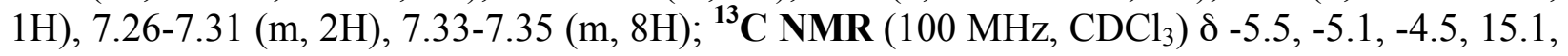
$17.6,17.9,18.2,25.7,25.9,33.6,39.1,63.6,72.9,73.3,73.4,73.7,75.4,76.3,77.6,88.5,127.5$, 127.7, 127.8, 128.3, 128.4, 138.3, 138.4; FTIR (thin film) $\square_{\max } 3561$ (br), 3066, 3032, 2956, 2929, 2857, 1497, 1472, 1463, 1455, 1389, 1361, 1256, 1106, 837, 776, 735, $697 \mathrm{~cm}^{-1}$; HRMS (ESI) calcd for $\mathrm{C}_{37} \mathrm{H}_{62} \mathrm{O}_{6} \mathrm{Si}_{2}[\mathrm{M}+\mathrm{Na}]^{+} 681.3983 \mathrm{~m} / \mathrm{z}$, found $681.3992 \mathrm{~m} / \mathrm{z}$. 

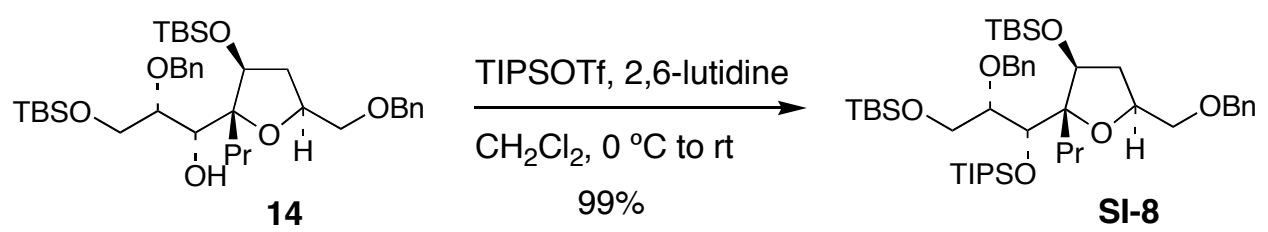

TIPS Ether SI-8. To a solution of alcohol $14(1.01 \mathrm{~g}, 1.54 \mathrm{mmol})$ in $\mathrm{CH}_{2} \mathrm{Cl}_{2}(3.1 \mathrm{~mL})$ at $0{ }^{\circ} \mathrm{C}$ was added 2,6-lutidine $(0.446 \mathrm{~mL}, 3.84 \mathrm{mmol})$ followed by TIPSOTf $(0.825 \mathrm{~mL}, 3.07$ $\mathrm{mmol}$ ) dropwise via syringe. The reaction mixture was stirred at room temperature for $24 \mathrm{~h}$, then was diluted with sat. aq. $\mathrm{NaHCO}_{3}$ and extracted with $\mathrm{Et}_{2} \mathrm{O}$. The combined organic layers were washed with brine, dried $\left(\mathrm{MgSO}_{4}\right)$, and concentrated in vacuo to yield the crude product. Purification of this residue by flash chromatography (1\% to 3\% EtOAc in hexanes) yielded the TIPS ether SI-8 (1.24 g, $1.52 \mathrm{mmol}, 99 \%)$ as a clear, light yellow oil. Data for SI-8: [D] ${ }_{\mathbf{D}}{ }^{\mathbf{2 4}}+3.0$ (c 3.9, $\left.\mathrm{CHCl}_{3}\right)$; ${ }^{1} \mathbf{H}$ NMR $\left(500 \mathrm{MHz}, \mathrm{CDCl}_{3}\right) \square 0.01(\mathrm{~s}, 3 \mathrm{H}), 0.02(\mathrm{~s}, 3 \mathrm{H}), 0.03(\mathrm{~s}, 3 \mathrm{H}), 0.04(\mathrm{~s}$, $3 \mathrm{H}), 0.80(\mathrm{t}, J=7.0 \mathrm{~Hz}, 3 \mathrm{H}), 0.87(\mathrm{~s}, 9 \mathrm{H}), 0.89(\mathrm{~s}, 9 \mathrm{H}), 1.04-1.08(\mathrm{~m}, 21 \mathrm{H}), 1.35-1.45(\mathrm{~m}, 1 \mathrm{H})$, $1.48-1.61(\mathrm{~m}, 2 \mathrm{H}), 1.63-1.76(\mathrm{~m}, 2 \mathrm{H}), 2.17(\mathrm{dt}, J=12.5,6.5 \mathrm{~Hz}, 1 \mathrm{H}), 3.44(\mathrm{dd}, J=10.0,5.5 \mathrm{~Hz}$, $1 \mathrm{H}), 3.56(\mathrm{dd}, J=10.0,6.5 \mathrm{~Hz}, 1 \mathrm{H}), 3.74(\mathrm{dt}, J=7.0,4.0 \mathrm{~Hz}, 1 \mathrm{H}), 3.86(\mathrm{dd}, J=11.0,7.0 \mathrm{~Hz}$, $1 \mathrm{H}), 3.93$ (dd, $J=11.0,3.5 \mathrm{~Hz}, 1 \mathrm{H}), 3.99(\mathrm{~d}, J=4.5 \mathrm{~Hz}, 1 \mathrm{H}), 4.17-4.23(\mathrm{~m}, 1 \mathrm{H}), 4.54$ (AB, $J=$ $12.0 \mathrm{~Hz}, 1 \mathrm{H}), 4.57(\mathrm{AB}, J=12.0 \mathrm{~Hz}, 1 \mathrm{H}), 4.64(\mathrm{~d}, J=12.0 \mathrm{~Hz}, 1 \mathrm{H}), 4.69(\mathrm{t}, J=7.0 \mathrm{~Hz}, 1 \mathrm{H})$, $4.78(\mathrm{~d}, J=11.5 \mathrm{~Hz}, 1 \mathrm{H}), 7.21-7.35(\mathrm{~m}, 10 \mathrm{H}) ;{ }^{13} \mathbf{C}$ NMR $\left(100 \mathrm{MHz}, \mathrm{CDCl}_{3}\right) \square-5.4,-5.3,-4.9$, -3.8, 13.4, 14.9, 17.9, 18.08, 18.13, 18.4, 18.5, 25.9, 35.6, 38.6, 63.7, 73.1, 73.2, 73.5, 74.5, 75.4, 75.5, 83.1, 90.1, 127.1, 127.4, 127.6, 128.0, 128.3, 138.6, 139.3; FTIR (thin film) $\square_{\max } 3089$, 3065, 3031, 2955, 2930, 2866, 1471, 1464, 1388, 1361, 1256, 1099, 837, 775, 733, 696, $678 \mathrm{~cm}^{-}$ ${ }^{1}$; HRMS (ESI) calcd for $\mathrm{C}_{46} \mathrm{H}_{82} \mathrm{O}_{6} \mathrm{Si}_{3}[\mathrm{M}+\mathrm{Na}]^{+} 837.5317 \mathrm{~m} / z$, found $837.5344 \mathrm{~m} / \mathrm{z}$.
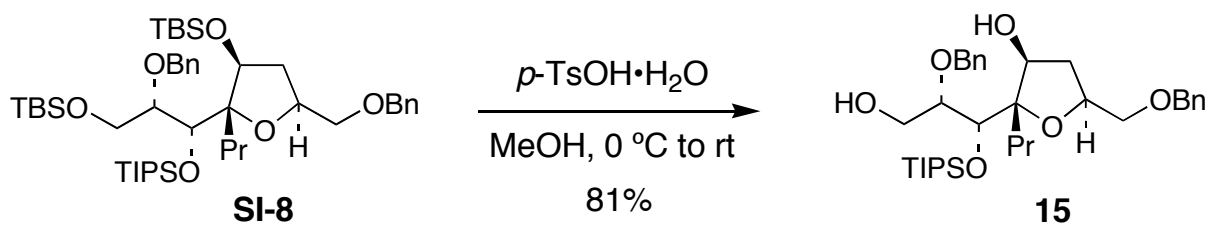

Diol 15. To a solution of TIPS ether SI-8 $(2.00 \mathrm{~g}, 2.46 \mathrm{mmol})$ in $\mathrm{MeOH}(48 \mathrm{~mL})$ at $0{ }^{\circ} \mathrm{C}$ was added $p-\mathrm{TsOH} \cdot \mathrm{H}_{2} \mathrm{O}(468 \mathrm{mg}, 2.46 \mathrm{mmol})$. The mixture was allowed to warm to room temperature and was stirred at this temperature for $36 \mathrm{~h}$, at which point TLC analysis indicated that both TBS ethers had been cleaved. The reaction was quenched by the addition of solid $\mathrm{NaHCO}_{3}(620 \mathrm{mg})$ and the resulting mixture was vigorously stirred for $15 \mathrm{~min}$. The mixture was filtered and concentrated in vacuo, and the resulting residue was partitioned in $\mathrm{Et}_{2} \mathrm{O}$ and $\mathrm{H}_{2} \mathrm{O}$. The layers were separated, and the aqueous layer was extracted with $\mathrm{Et}_{2} \mathrm{O}$. The combined organic layers were washed with brine, dried $\left(\mathrm{MgSO}_{4}\right)$, and concentrated in vacuo. Purification of the residue by flash chromatography (20\% EtOAc in hexanes) provided diol 15 (1.16 g, 1.98 mmol, 81\%) as a clear, colorless oil. Data for 15: [0] $\left.\right|_{\mathbf{D}}{ }^{24}-3.0\left(\right.$ c 3.9, $\left.\mathrm{CHCl}_{3}\right) ;{ }^{1} \mathbf{H}$ NMR $(500$ $\left.\mathrm{MHz}, \mathrm{CDCl}_{3}\right) \square 0.85(\mathrm{t}, \mathrm{J}=7.0 \mathrm{~Hz}, 3 \mathrm{H}), 1.04-1.11(\mathrm{~m}, 21 \mathrm{H}), 1.26-1.36(\mathrm{~m}, 1 \mathrm{H}), 1.50-1.63(\mathrm{~m}$, $3 \mathrm{H}), 1.82(\mathrm{dt}, J=12.5,8.5 \mathrm{~Hz}, 1 \mathrm{H}), 2.34$ (dt, $J=12.5,7.0 \mathrm{~Hz}, 1 \mathrm{H}), 2.48$ (br s, Oㅂ), 3.45 (dd, $J=$ $10.0,5.0 \mathrm{~Hz}, 1 \mathrm{H}), 3.52(\mathrm{dd}, J=10.0,4.0 \mathrm{~Hz}, 1 \mathrm{H}), 3.54(\mathrm{~d}, J=4.5 \mathrm{~Hz}, \mathrm{OH}), 3.60-3.64(\mathrm{~m}, 1 \mathrm{H})$, $3.86(\mathrm{dt}, J=12.0,5.0 \mathrm{~Hz}, 1 \mathrm{H}), 4.00(\mathrm{ddd}, J=12.0,6.0,3.0 \mathrm{~Hz}, 1 \mathrm{H}), 4.05(\mathrm{~d}, J=4.0 \mathrm{~Hz}, 1 \mathrm{H})$, 
4.14-4.20 (m, 1H), $4.55(\mathrm{~d}, J=11.5 \mathrm{~Hz}, 1 \mathrm{H}), 4.57(\mathrm{br} \mathrm{s}, 1 \mathrm{H}), 4.61(\mathrm{td}, J=8.0,4.5 \mathrm{~Hz}, 1 \mathrm{H}), 4.77$ $(\mathrm{d}, J=11.5 \mathrm{~Hz}, 1 \mathrm{H}), 7.26-7.37(\mathrm{~m}, 10 \mathrm{H}) ;{ }^{13} \mathbf{C} \mathbf{N M R}\left(125 \mathrm{MHz}, \mathrm{CDCl}_{3}\right) \square 13.7,15.0,16.8,18.39$, $18.40,34.4,35.9,60.7,71.2,73.3,73.5,75.1,75.9,80.3,88.3,127.56,127.58,127.9,128.0$, 128.3, 128.5, 137.6, 138.1; FTIR (thin film) $\square_{\max } 3424$ (br), 3065, 3031, 2944, 2867, 1496, 1454, 1358, 1092, 1068, 883, 736, 697, $679 \mathrm{~cm}^{-1}$; HRMS (ESI) calcd for $\mathrm{C}_{34} \mathrm{H}_{54} \mathrm{O}_{6} \mathrm{Si}[\mathrm{M}+\mathrm{Na}]^{+}$ $609.3587 \mathrm{~m} / \mathrm{z}$, found $609.3585 \mathrm{~m} / \mathrm{z}$.

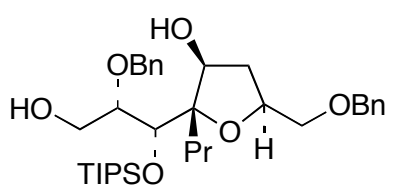

15

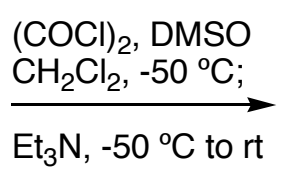

$\mathrm{Et}_{3} \mathrm{~N},-50^{\circ} \mathrm{C}$ to $\mathrm{rt}$

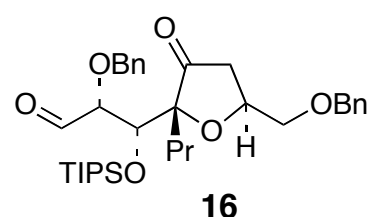

16

Ketoaldehyde 16. To a solution of DMSO $(0.051 \mathrm{~mL}, 0.72 \mathrm{mmol})$ in $\mathrm{CH}_{2} \mathrm{Cl}_{2}(0.3 \mathrm{~mL})$ at $-50{ }^{\circ} \mathrm{C}$ was added $(\mathrm{COCl})_{2}\left(0.18 \mathrm{~mL}\right.$ of a $2 \mathrm{M}$ soln. in $\left.\mathrm{CH}_{2} \mathrm{Cl}_{2}, 0.36 \mathrm{mmol}\right)$ dropwise via syringe. This mixture was stirred for $10 \mathrm{~min}$, then a solution of diol 15 (35 $\mathrm{mg}, 0.060 \mathrm{mmol})$ in $\mathrm{CH}_{2} \mathrm{Cl}_{2}(0.3 \mathrm{~mL})$ was added via cannula. The resulting cloudy mixture was stirred at $-50{ }^{\circ} \mathrm{C}$ for $1 \mathrm{~h}$, and $\mathrm{Et}_{3} \mathrm{~N}(0.15 \mathrm{~mL}, 1.1 \mathrm{mmol})$ was added. The mixture was warmed to room temperature and partitioned in $\mathrm{Et}_{2} \mathrm{O}$ and $\mathrm{H}_{2} \mathrm{O}$. The layers were separated, the aqueous layer was extracted with $\mathrm{Et}_{2} \mathrm{O}$, and the combined organic layers were washed with brine, dried $\left(\mathrm{Na}_{2} \mathrm{SO}_{4}\right)$, and concentrated in vacuo. The crude ketoaldehyde $\mathbf{1 6}$ so obtained was used directly in the next transformation without further purification. A sample of $\mathbf{1 6}$ for characterization could be obtained (ca. 90\% purity) by flash chromatography (10\% EtOAc in hexanes). Data for 16: $\left[\mathrm{ID}_{\mathbf{D}}{ }^{24}-50\left(\mathrm{c} 2.0, \mathrm{CHCl}_{3}\right) ;{ }^{1} \mathbf{H}\right.$ NMR $\left(500 \mathrm{MHz}, \mathrm{CDCl}_{3}\right) \square 0.83(\mathrm{t}, J=8.5 \mathrm{~Hz}, 3 \mathrm{H}), 1.00-1.05(\mathrm{~m}$, $21 \mathrm{H}), 1.15-1.30(\mathrm{~m}, 1 \mathrm{H}), 1.47(\mathrm{td}, J=16.0,4.5 \mathrm{~Hz}, 1 \mathrm{H}), 1.52-1.62(\mathrm{~m}, 1 \mathrm{H}), 1.90(\mathrm{td}, J=16.0$, $4.0 \mathrm{~Hz}, 1 \mathrm{H}), 2.31\left(\underline{\mathrm{ABX}}, J_{\mathrm{AB}}=23.0 \mathrm{~Hz}, J_{\mathrm{AX}}=11.7 \mathrm{~Hz}, 1 \mathrm{H}\right), 2.39\left(\mathrm{ABX}, J_{\mathrm{AB}}=23.0 \mathrm{~Hz}, J_{\mathrm{BX}}=\right.$ $9.3 \mathrm{~Hz}, 1 \mathrm{H}), 3.54\left(\underline{\mathrm{ABX}}, J_{\mathrm{AB}}=13.0 \mathrm{~Hz}, J_{\mathrm{AX}}=5.7 \mathrm{~Hz}, 1 \mathrm{H}\right), 3.56 \mathrm{AB}, \underline{\mathrm{B}}, J_{\mathrm{AB}}=13.0 \mathrm{~Hz}, J_{\mathrm{BX}}=4.3$ $\mathrm{Hz}, 1 \mathrm{H}), 3.71(\mathrm{~d}, J=7.5 \mathrm{~Hz}, 1 \mathrm{H}), 4.33(\mathrm{ddt}, J=11.5,9.5,5.0 \mathrm{~Hz}, 1 \mathrm{H}), 4.40(\mathrm{~d}, J=7.5 \mathrm{~Hz}, 1 \mathrm{H})$, $4.50(\underline{\mathrm{AB}}, J=14.5 \mathrm{~Hz}, 1 \mathrm{H}), 4.52(\mathrm{~d}, J=14.5 \mathrm{~Hz}, 1 \mathrm{H}), 4.58(\mathrm{AB}, J=15.0 \mathrm{~Hz}, 1 \mathrm{H}), 4.67(\mathrm{~d}, J=$ $14.5 \mathrm{~Hz}, 1 \mathrm{H}), 7.24-7.37(\mathrm{~m}, 10 \mathrm{H}), 10.0$ (as, $1 \mathrm{H}) ;{ }^{13} \mathbf{C} \mathbf{N M R}\left(100 \mathrm{MHz}, \mathrm{CDCl}_{3}\right) \square 12.4,12.7$, $14.3,16.5,18.0,18.10,18.13,37.5,38.6,71.8,72.4,72.8,73.1,78.6,80.5,87.7,127.5,127.6$, 128.2, 128.25, 128.27, 128.5, 136.7, 138.1, 198.3, 214.9; FTIR (thin film) $\square_{\max } 3065,3032$, 2946, 2868, 1753, 1727, 1588, 1497, 1455, 1363, 1096, 1013, 882, 795, 739, 698, $680 \mathrm{~cm}^{-1}$; HRMS (ESI) calcd for $\mathrm{C}_{34} \mathrm{H}_{50} \mathrm{O}_{6} \mathrm{Si}[\mathrm{M}+\mathrm{Na}]^{+} 605.3274 \mathrm{~m} / \mathrm{z}$, found $605.3278 \mathrm{~m} / \mathrm{z}$. 


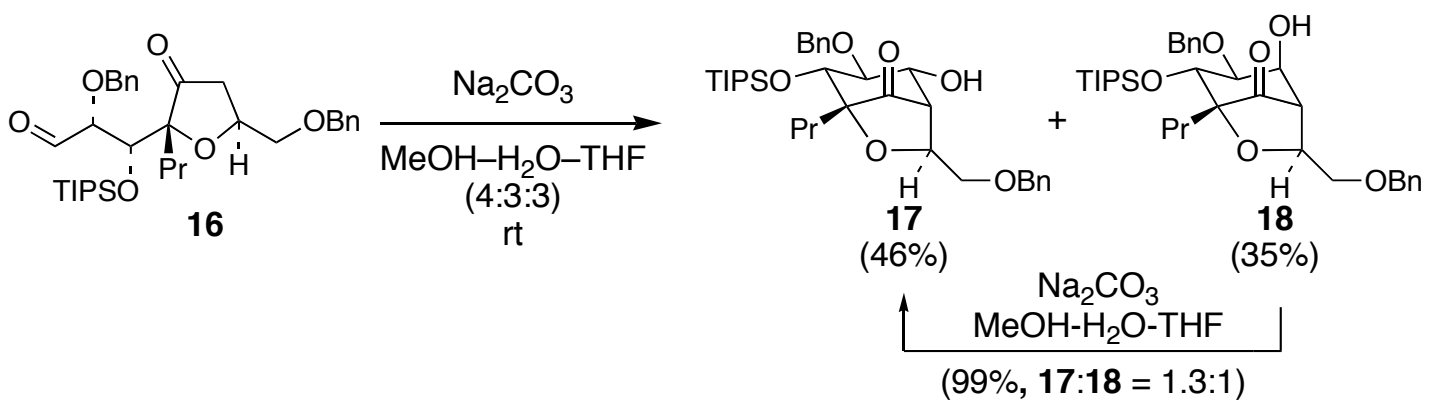

Bicyclic Alcohols 17 and 18. To a vigorously stirred solution of ketoaldehyde $\mathbf{1 6}$ (crude from above) in a $4: 3$ mixture of $\mathrm{MeOH}: \mathrm{H}_{2} \mathrm{O}(2.1 \mathrm{~mL})$ at room temperature was added $\mathrm{Na}_{2} \mathrm{CO}_{3} \cdot 10 \mathrm{H}_{2} \mathrm{O}(13 \mathrm{mg}, 0.045 \mathrm{mmol})$. THF $(1.5 \mathrm{~mL})$ was added to solubilize a small amount of material that had separated as an oil. The mixture was stirred for $1 \mathrm{~h}$, then sat. aq. $\mathrm{NaHCO}_{3}$ was added and the mixture was extracted with $\mathrm{Et}_{2} \mathrm{O}$. The combined organic layers were washed with brine, dried $\left(\mathrm{MgSO}_{4}\right)$, and concentrated in vacuo. The resultant residue was purified by flash chromatography (10\% to 20\% EtOAc in hexanes) to afford alcohol $17(16 \mathrm{mg}, 0.027 \mathrm{mmol}$, $46 \%)$ along with its diastereomer $18(12 \mathrm{mg}, 0.021 \mathrm{mmol}, 35 \%)$ as clear oils, the latter of which solidified upon drying in vacuo. The minor diastereomer $\mathbf{1 8}$ was resubjected to the reaction conditions to give a $1.3: 1$ ( ${ }^{1} \mathrm{H}$ NMR analysis) thermodynamic ratio of $\mathbf{1 7}$ and $\mathbf{1 8}$. Compound $\mathbf{1 7}$ could thus be obtained in an overall yield of $71 \%$ after two recycles of 18 . Data for 17: []$_{\mathbf{D}}{ }^{24}$ $+31\left(c 1.9, \mathrm{CHCl}_{3}\right) ;{ }^{1} \mathbf{H}$ NMR $\left(500 \mathrm{MHz}, \mathrm{CDCl}_{3}\right) \square 0.86(\mathrm{t}, J=7.5 \mathrm{~Hz}, 3 \mathrm{H}), 1.00-1.09(\mathrm{~m}, 21 \mathrm{H})$, $1.11-1.21(\mathrm{~m}, 1 \mathrm{H}), 1.31-1.42(\mathrm{~m}, 1 \mathrm{H}), 1.58$ (ddd, $J=14.0,12.0,5.0 \mathrm{~Hz}, 1 \mathrm{H}), 1.81$ (d, $J=3.5 \mathrm{~Hz}$, $1 \mathrm{H}), 1.87$ (ddd, $J=14.0,12.0,4.0 \mathrm{~Hz}, 1 \mathrm{H}), 2.63(\mathrm{~d}, J=3.5 \mathrm{~Hz}, 1 \mathrm{H}), 3.45\left(\underline{\mathrm{ABX}}, J_{\mathrm{AB}}=10.0 \mathrm{~Hz}\right.$, $\left.J_{\mathrm{AX}}=4.0 \mathrm{~Hz}, 1 \mathrm{H}\right), 3.48\left(\mathrm{ABX}, J_{\mathrm{AB}}=10.0 \mathrm{~Hz}, J_{\mathrm{BX}}=4.0 \mathrm{~Hz}, 1 \mathrm{H}\right), 3.56(\mathrm{~d}, J=7.5 \mathrm{~Hz}, 1 \mathrm{H}), 3.79-$ $3.86(\mathrm{~m}, 2 \mathrm{H}), 4.43(\mathrm{~d}, J=12.5 \mathrm{~Hz}, 1 \mathrm{H}), 4.51(\mathrm{~d}, 12.0 \mathrm{~Hz}, 1 \mathrm{H}), 4.55(\mathrm{t}, J=4.0 \mathrm{~Hz}, 1 \mathrm{H}), 4.77(\mathrm{~d}, J$ $=12.0 \mathrm{~Hz}, 1 \mathrm{H}), 4.90(\mathrm{~d}, J=12.0 \mathrm{~Hz}, 1 \mathrm{H}), 7.23-7.30(\mathrm{~m}, 2 \mathrm{H}), 7.30-7.36(\mathrm{~m}, 8 \mathrm{H}) ;{ }^{13} \mathbf{C}$ NMR $(100$ $\left.\mathrm{MHz}, \mathrm{CDCl}_{3}\right) \square 13.5,14.6,16.2,18.2,18.4,30.7,54.8,72.2,72.6,72.7,73.4,74.5,79.3,86.0$, 86.8, 127.1, 127.6, 127.6, 127.7, 128.3, 128.4, 137.6, 139.1, 209.0; FTIR (thin film) $\square \max 3451$ (br), 3065, 3031, 2944, 2867, 1767, 1454, 1363, 1114, 1050, 884, 818, 755, 737, 697, $682 \mathrm{~cm}^{-1}$; HRMS (ESI) calcd for $\mathrm{C}_{34} \mathrm{H}_{50} \mathrm{O}_{6} \mathrm{Si}[\mathrm{M}+\mathrm{Na}]^{+} 605.3274 \mathrm{~m} / \mathrm{z}$, found $605.3278 \mathrm{~m} / \mathrm{z}$.

Data for 18: mp 106-109 ${ }^{\circ} \mathrm{C}$; [ [D] $\mathbf{l}^{24}+35\left(c\right.$ 1.5, $\left.\mathrm{CHCl}_{3}\right) ;{ }^{1} \mathbf{H}$ NMR $\left(500 \mathrm{MHz}, \mathrm{CDCl}_{3}\right)$ $\square 0.87(\mathrm{t}, J=7.5 \mathrm{~Hz}, 3 \mathrm{H}), 1.01-1.11(\mathrm{~m}, 21 \mathrm{H}), 1.12-1.23(\mathrm{~m}, 1 \mathrm{H}), 1.23-1.38(\mathrm{~m}, 1 \mathrm{H}), 1.61$ (ddd, $J=14.0,11.5,4.5 \mathrm{~Hz}, 1 \mathrm{H}), 1.90(\mathrm{ddd}, J=14.0,12.5,4.5 \mathrm{~Hz}, 1 \mathrm{H}), 2.61(\mathrm{~d}, 0.5 \mathrm{~Hz}, 1 \mathrm{H}), 2.87$ (d, $J=5.0 \mathrm{~Hz}, \mathrm{OH}), 3.34(\mathrm{dd}, J=10.0,6.0 \mathrm{~Hz}, 1 \mathrm{H}), 3.49(\mathrm{dd}, J=9.5,4.5 \mathrm{~Hz}, 1 \mathrm{H}), 3.79(J=8.5 \mathrm{~Hz}$, $1 \mathrm{H}), 3.85(\mathrm{dd}, J=8.5,4.5 \mathrm{~Hz}, 1 \mathrm{H}), 4.08(\mathrm{dd}, J=6.0,4.5 \mathrm{~Hz}, 1 \mathrm{H}), 4.19$ (at, $J=5.0 \mathrm{~Hz}, 1 \mathrm{H}), 4.44$ $(\mathrm{d}, J=12.0 \mathrm{~Hz}, 1 \mathrm{H}), 4.52(\mathrm{~d}, J=12.0 \mathrm{~Hz}, 1 \mathrm{H}), 4.58(\underline{\mathrm{AB}}, J=11.0 \mathrm{~Hz}, 1 \mathrm{H}), 4.63(\mathrm{AB}, J=11.5$ $\mathrm{Hz}, 1 \mathrm{H}), 7.25-7.38(\mathrm{~m}, 10 \mathrm{H}) ;{ }^{13} \mathbf{C}$ NMR $\left(100 \mathrm{MHz}, \mathrm{CDCl}_{3}\right) \square 13.3,13.5,14.5,16.1,18.2,18.4$, $29.7,30.4,51.3,54.8,67.1,71.6,72.7,73.0,73.5,79.5,82.8,85.3,127.1,127.6,127.7,127.8$, $128.2,128.3,128.4,128.7,137.3,137.5,207.0$; FTIR (thin film) $\square_{\max } 3467,3090,3065,3032$, 2961, 2944, 2866, 1771, 1497, 1465, 1455, 1367, 1256, 1111, 883, 739, 698, $682 \mathrm{~cm}^{-1}$; HRMS (ESI) calcd for $\mathrm{C}_{34} \mathrm{H}_{50} \mathrm{O}_{6} \mathrm{Si}[\mathrm{M}+\mathrm{Na}]^{+} 605.3274 \mathrm{~m} / \mathrm{z}$, found $605.3278 \mathrm{~m} / \mathrm{z}$.

Selected ${ }^{1} \mathrm{H}$ NMR coupling constants and ${ }^{1} \mathrm{H}$ nOe data for $\mathbf{1 7}$ and $\mathbf{1 8}$ : 


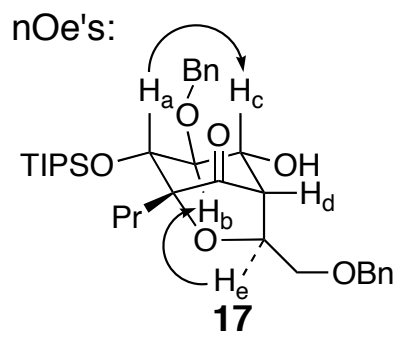

nOe's:
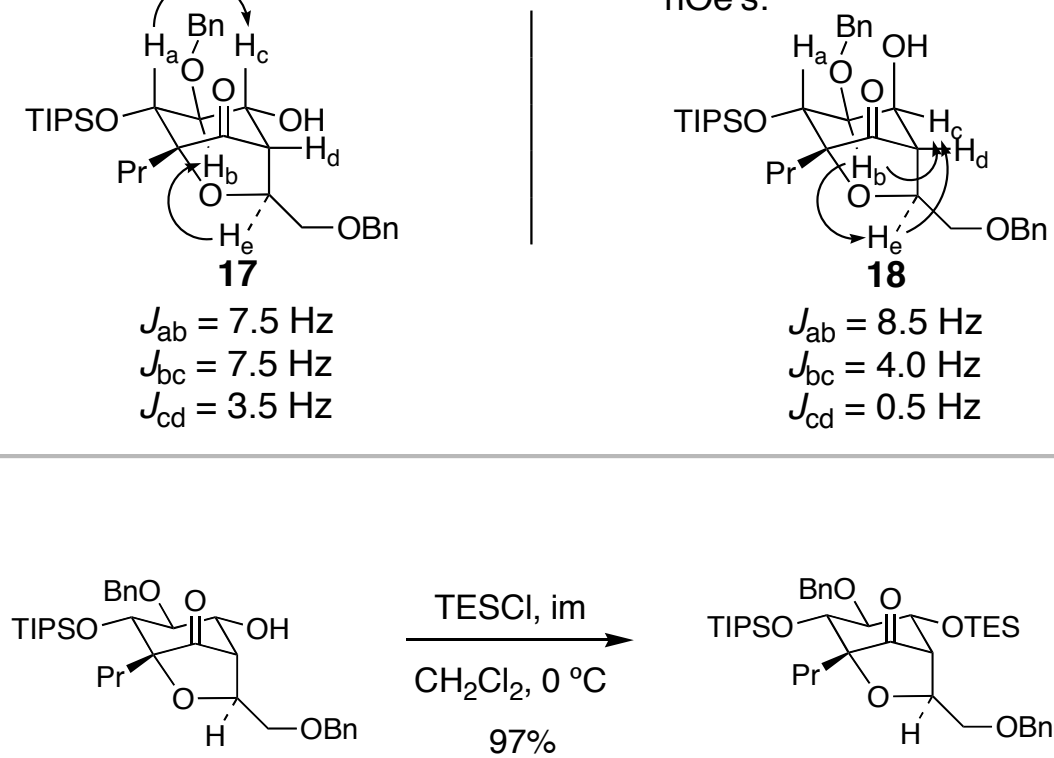

17
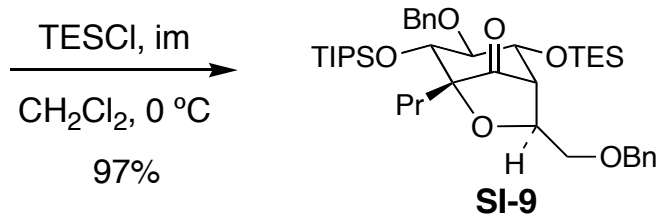

TES Ether SI-9. To a solution of alcohol $17(452 \mathrm{mg}, 0.777 \mathrm{mmol})$ in $\mathrm{CH}_{2} \mathrm{Cl}_{2}(3.9 \mathrm{~mL})$ at room temperature was added imidazole $(159 \mathrm{mg}, 2.33 \mathrm{mmol})$ followed by TESCl $(0.260 \mathrm{~mL}$, $1.55 \mathrm{mmol})$. The reaction mixture was stirred at room temperature for $30 \mathrm{~min}$, at which point $\mathrm{H}_{2} \mathrm{O}$ was added. The mixture was extracted with $\mathrm{Et}_{2} \mathrm{O}$, and the combined organic layers were washed with brine, dried $\left(\mathrm{MgSO}_{4}\right)$, and concentrated in vacuo. Purification of the residue by flash chromatography (5\% EtOAc in hexanes) afforded the TES ether SI-9 (524 mg, 0.753 mmol, 97\%) as a colorless oil. Data for SI-9: [D] ${ }_{\mathbf{D}}{ }^{24}+36$ (c 2.1, $\left.\mathrm{CHCl}_{3}\right) ;{ }^{\mathbf{1}} \mathbf{H} \mathbf{~ N M R}(500 \mathrm{MHz}$, $\left.\mathrm{CDCl}_{3}\right) \square 0.51-0.57(\mathrm{~m}, 6 \mathrm{H}), 0.86(\mathrm{t}, J=7.5 \mathrm{~Hz}, 3 \mathrm{H}), 0.87(\mathrm{t}, J=8.0 \mathrm{~Hz}, 9 \mathrm{H}), 0.92-1.03(\mathrm{~m}$, $21 \mathrm{H}), 1.11-1.22(\mathrm{~m}, 1 \mathrm{H}), 1.32-1.43(\mathrm{~m}, 1 \mathrm{H}), 1.57$ (ddd, $J=14.0,12.0,5.0 \mathrm{~Hz}, 1 \mathrm{H}), 1.85$ (ddd, $J$ $=14.0,12.5,4.5 \mathrm{~Hz}, 1 \mathrm{H}), 2.60(\mathrm{~d}, J=4.0 \mathrm{~Hz}, 1 \mathrm{H}), 3.44(\mathrm{dd}, J=10.0,3.5 \mathrm{~Hz}, 1 \mathrm{H}), 3.48(\mathrm{~d}, J=$ $8.0 \mathrm{~Hz}, 1 \mathrm{H}$ ), 3.50 (dd, $J=10.0,4.5 \mathrm{~Hz}, 1 \mathrm{H}), 3.82$ (at, $J=8.0 \mathrm{~Hz}, 1 \mathrm{H}), 3.98$ (dd, $J=7.5,4.0 \mathrm{~Hz}$, $1 \mathrm{H}), 4.43(\mathrm{~d}, J=12.0 \mathrm{~Hz}, 1 \mathrm{H}), 4.53(\mathrm{~d}, J=12.0 \mathrm{~Hz}, 1 \mathrm{H}), 4.55(\mathrm{~d}, J=12.0 \mathrm{~Hz}, 1 \mathrm{H}), 4.60(\mathrm{t}, J=$ $4.0 \mathrm{~Hz}, 1 \mathrm{H}), 5.18(\mathrm{~d}, J=11.5 \mathrm{~Hz}, 1 \mathrm{H}), 7.19-7.24(\mathrm{~m}, 1 \mathrm{H}), 7.24-7.30(\mathrm{~m}, 7 \mathrm{H}), 7.30-7.35(\mathrm{~m}, 2 \mathrm{H})$; ${ }^{13}$ C NMR (125 MHz, $\left.\mathrm{CDCl}_{3}\right) \square 4.9,6.7,13.4,14.6,16.2,18.2,18.4,30.7,55.7,72.2,72.7,73.3$, 74.4, 74.5, 78.9, 86.1, 86.7, 126.4, 126.8, 127.6, 127.7, 127.9, 128.3, 137.7, 139.0, 209.4; FTIR (thin film) $\square_{\max } 3031,2958,2869,1768,1455,1367,1241,1090,1015,776,731 \mathrm{~cm}^{-1}$; HRMS (ESI) calcd for $\mathrm{C}_{40} \mathrm{H}_{64} \mathrm{O}_{6} \mathrm{Si}_{2}[\mathrm{M}+\mathrm{Na}]^{+} 719.4139 \mathrm{~m} / \mathrm{z}$, found $719.4144 \mathrm{~m} / \mathrm{z}$.
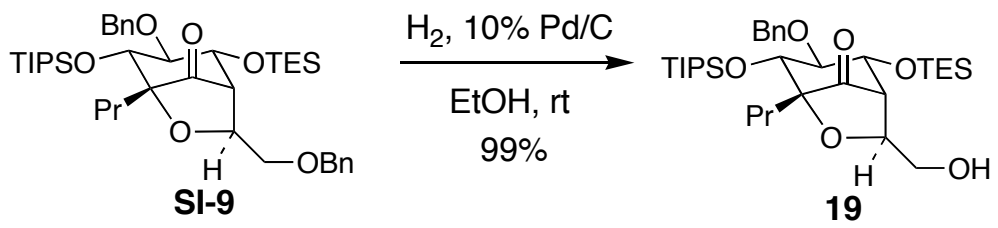

Alcohol 19. To a solution of benzyl ether SI-9 $(21 \mathrm{mg}, 0.030 \mathrm{mmol})$ in EtOH $(0.6 \mathrm{~mL})$ was added $10 \% \mathrm{Pd} / \mathrm{C}(6 \mathrm{mg})$ in one portion. The mixture was stirred at room temperature under 
an atmosphere of $\mathrm{H}_{2}(1 \mathrm{~atm})$ for $36 \mathrm{~h}$. Additional portions of $\mathrm{Pd} / \mathrm{C}(6 \mathrm{mg})$ were added at regular intervals during this time. The reaction mixture was evacuated, then opened to the atmosphere and diluted with $\mathrm{Et}_{2} \mathrm{O}$. Filtration of the slurry through a plug of Celite and concentration of the filtrate in vacuo afforded alcohol $19(18 \mathrm{mg}, 0.030 \mathrm{mmol}, 99 \%)$ as a clear oil which solidified upon standing at room temperature. Data for 19: mp 60-62 ${ }^{\circ} \mathrm{C}$; [ [ $]_{\mathbf{D}}{ }^{\mathbf{2 4}}+37$ (c 3.6, $\left.\mathrm{CHCl}_{3}\right) ;{ }^{1} \mathbf{H}$ NMR $\left(500 \mathrm{MHz}, \mathrm{CDCl}_{3}\right) \square$ 0.56-0.62 (m, 6H), 0.89-1.04 (m, 24H), $0.92(\mathrm{t}, J=8.0 \mathrm{~Hz}, 9 \mathrm{H})$, $1.16-1.28(\mathrm{~m}, 1 \mathrm{H}), 1.31-1.42(\mathrm{~m}, 1 \mathrm{H}), 1.60(\mathrm{ddd}, J=13.5,12.0,4.0 \mathrm{~Hz}, 1 \mathrm{H}), 1.86(\mathrm{dd}, J=7.5$, $5.0 \mathrm{~Hz}, \mathrm{OH}), 1.91(\mathrm{ddd}, J=14.0,13.0,4.5 \mathrm{~Hz}, 1 \mathrm{H}), 2.50(\mathrm{~d}, J=4.0 \mathrm{~Hz}, 1 \mathrm{H}), 3.49$ (ddd, $J=11.5$, $11.0,4.5 \mathrm{~Hz}, 1 \mathrm{H}), 3.51(\mathrm{~d}, J=8.0 \mathrm{~Hz}, 1 \mathrm{H}), 3.69$ (ddd, $J=11.0,7.5,3.5 \mathrm{~Hz}, 1 \mathrm{H}), 3.84$ (at, $J=8.0$ $\mathrm{Hz}, 1 \mathrm{H}), 4.00$ (dd, $J=7.0,4.0 \mathrm{~Hz}, 1 \mathrm{H}), 4.56$ (at, $J=4.5 \mathrm{~Hz}, 1 \mathrm{H}), 4.57$ (d, $J=11.5 \mathrm{~Hz}, 1 \mathrm{H}), 5.19$ $(\mathrm{d}, J=12.0 \mathrm{~Hz}, 1 \mathrm{H}), 7.20-7.25(\mathrm{~m}, 1 \mathrm{H}), 7.25-7.32(\mathrm{~m}, 4 \mathrm{H}) ;{ }^{13} \mathbf{C} \mathbf{N M R}\left(125 \mathrm{MHz}, \mathrm{CDCl}_{3}\right) \square 5.0$, $6.7,13.4,14.5,16.5,18.2,18.3,30.6,55.2,65.3,73.9,74.3,74.6,78.7,85.9,86.7,126.3,126.9$, 127.9, 138.9, 209.0; FTIR (thin film) $\square_{\max }$ 3480, 2958, 2869, 1768, 1465, 1375, 1241, 1136, $1088,1015,884,774,731,682 \mathrm{~cm}^{-1}$; HRMS (ESI) calcd for $\mathrm{C}_{33} \mathrm{H}_{58} \mathrm{O}_{6} \mathrm{Si}_{2}[\mathrm{M}+\mathrm{Na}]^{+} 629.3670$ $\mathrm{m} / \mathrm{z}$, found $629.3677 \mathrm{~m} / \mathrm{z}$.

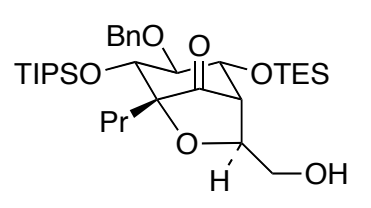

19

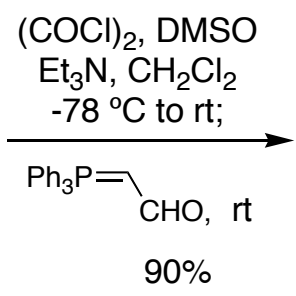

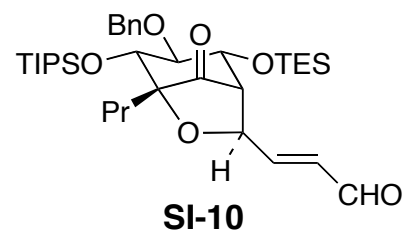

SI-10

Enal SI-10. To a solution of DMSO $(0.077 \mathrm{~mL}, 1.1 \mathrm{mmol})$ in $\mathrm{CH}_{2} \mathrm{Cl}_{2}(2.0 \mathrm{~mL})$ at $-78^{\circ} \mathrm{C}$ was added $(\mathrm{COCl})_{2}(0.069 \mathrm{~mL}, 0.54 \mathrm{mmol})$ dropwise via syringe. The mixture was stirred for 10 min at $-78{ }^{\circ} \mathrm{C}$, then a solution of alcohol $19(164 \mathrm{mg}, 0.271 \mathrm{mmol})$ in $\mathrm{CH}_{2} \mathrm{Cl}_{2}(1.0 \mathrm{~mL})$ was added via cannula. The resulting cloudy mixture was stirred at $-78^{\circ} \mathrm{C}$ for $1 \mathrm{~h}$, and $\mathrm{Et}_{3} \mathrm{~N}(0.19$ $\mathrm{mL}, 1.4 \mathrm{mmol}$ ) was added. The mixture was warmed to room temperature, and (triphenylphosphoranylidene)acetaldehye $(247 \mathrm{mg}, 1.36 \mathrm{mmol})$ was added. The resulting mixture was stirred for $30 \mathrm{~min}$ at room temperature, then $\mathrm{Et}_{2} \mathrm{O}$ was added and the mixture was filtered through a plug of Celite. Concentration of the filtrate gave an orange solid, which was purified by flash chromatography (5\% to 10\% EtOAc in hexanes) to afford enal SI-10 (154 mg, $0.244 \mathrm{mmol}, 90 \%)$ as a yellow oil. Data for SI-10: [D] $]_{\mathbf{D}}{ }^{\mathbf{4}}+68\left(\mathrm{c} \mathrm{3.3}, \mathrm{CHCl}_{3}\right) ;{ }^{\mathbf{1}} \mathbf{H}$ NMR $(500$ $\left.\mathrm{MHz}_{\mathrm{CDCl}}\right) \square 0.56-0.62(\mathrm{~m}, 6 \mathrm{H}), 0.92$ (t, $\left.J=8.0 \mathrm{~Hz}, 9 \mathrm{H}\right), 0.93(\mathrm{t}, J=7.0 \mathrm{~Hz}, 3 \mathrm{H}), 0.95-1.04$ $(\mathrm{m}, 21 \mathrm{H}), 1.13-1.20(\mathrm{~m}, 1 \mathrm{H}), 1.34-1.42(\mathrm{~m}, 1 \mathrm{H}), 1.61(\mathrm{ddd}, J=14.0,12.0,4.5 \mathrm{~Hz}, 1 \mathrm{H}), 1.91$ $(\mathrm{ddd}, J=14.0,12.5,4.5 \mathrm{~Hz}, 1 \mathrm{H}), 2.59(\mathrm{~d}, J=4.0 \mathrm{~Hz}, 1 \mathrm{H}), 3.55(\mathrm{~d}, J=8.0 \mathrm{~Hz}, 1 \mathrm{H}), 3.88$ (at, $J=$ $7.5 \mathrm{~Hz}, 1 \mathrm{H}), 4.03$ (dd, $J=8.0,4.0 \mathrm{~Hz}, 1 \mathrm{H}), 4.59$ (d, $J=12.0 \mathrm{~Hz}, 1 \mathrm{H}), 5.17-5.22(\mathrm{~m}, 2 \mathrm{H}), 6.28$ $(\mathrm{ddd}, J=16.0,7.5,1.5 \mathrm{~Hz}, 1 \mathrm{H}), 6.59(\mathrm{dd}, J=16.0,6.0 \mathrm{~Hz}, 1 \mathrm{H}), 7.21-7.33(\mathrm{~m}, 5 \mathrm{H}), 9.57(\mathrm{~d}, J=$ $7.5 \mathrm{~Hz}, 1 \mathrm{H}) ;{ }^{13} \mathrm{C}$ NMR $\left(100 \mathrm{MHz}, \mathrm{CDCl}_{3}\right) \square 4.9,6.7,13.5,14.5,16.8,18.1,18.3,29.7,30.5$, 57.7, 72.4, 74.2, 74.7, 78.6, 86.1, 86.7, 126.3, 127.0, 128.0, 132.3, 138.7, 153.2, 192.8, 208.1; FTIR (thin film) $\square_{\max } 2959,2869,1766,1699,1465,1370,1092,1015,777,732 \mathrm{~cm}^{-1}$; HRMS (ESI) calcd for $\mathrm{C}_{35} \mathrm{H}_{58} \mathrm{O}_{6} \mathrm{Si}_{2}[\mathrm{M}+\mathrm{Na}]^{+} 653.3670 \mathrm{~m} / \mathrm{z}$, found $653.3688 \mathrm{~m} / \mathrm{z}$. 


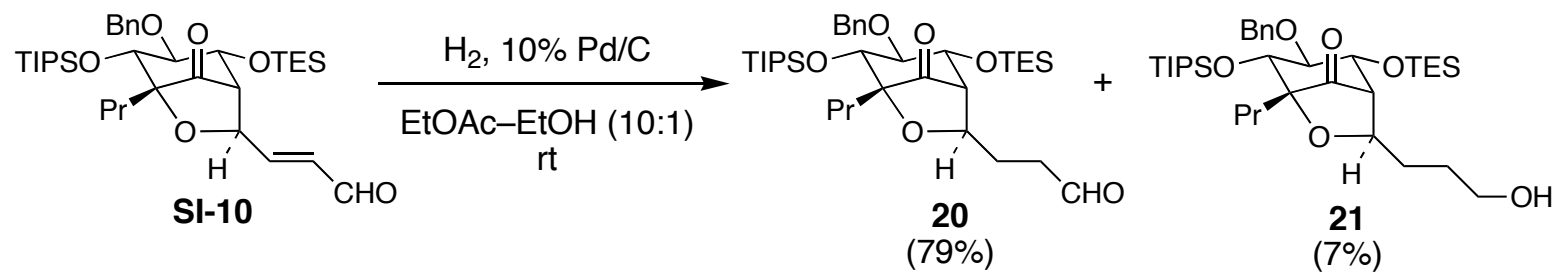

Aldehyde 20. Enal SI-10 (26 mg, $0.042 \mathrm{mmol})$ was dissolved in 10:1 EtOAc-EtOH $(0.92 \mathrm{~mL})$, and $10 \% \mathrm{Pd} / \mathrm{C}(10 \mathrm{mg})$ was added in one portion. The mixture was stirred at room temperature under an atmosphere of $\mathrm{H}_{2}(1 \mathrm{~atm})$ for $1 \mathrm{~h}$, a second portion of $\mathrm{Pd} / \mathrm{C}(10 \mathrm{mg}) \mathrm{was}$ added, and the mixture was stirred for an additional $1 \mathrm{~h}$. The reaction mixture was then diluted with $\mathrm{Et}_{2} \mathrm{O}$, filtered through a pad of Celite, and concentrated in vacuo. Purification of the residue by flash chromatography (5\% to $10 \%$ to $20 \%$ EtOAc) afforded aldehyde 20 (21 $\mathrm{mg}, 0.033 \mathrm{mmol}$, 79\%) along with alcohol 21 (2 mg, $0.003 \mathrm{mmol}, 7 \%)$. Data for 20: [D] ${ }_{\mathbf{D}}{ }^{24}+53$ (c 2.1, $\left.\mathrm{CHCl}_{3}\right){ }^{1} \mathbf{H}$ NMR $\left(500 \mathrm{MHz}, \mathrm{CDCl}_{3}\right) \square 0.55(\mathrm{~m}, 6 \mathrm{H}), 0.91(\mathrm{t}, J=7.5 \mathrm{~Hz}, 3 \mathrm{H}), 0.92(\mathrm{t}, J=8.0 \mathrm{~Hz}, 9 \mathrm{H}), 0.94-$ $1.04(\mathrm{~m}, 21 \mathrm{H}), 1.08-1.19(\mathrm{~m}, 1 \mathrm{H}), 1.21-1.30(\mathrm{~m}, 1 \mathrm{H}), 1.50-1.58(\mathrm{~m}, 1 \mathrm{H}) ; 1.58-1.65(\mathrm{~m}, 1 \mathrm{H})$, $1.66-1.74(\mathrm{~m}, 1 \mathrm{H}), 1.83$ (ddd, $J=14.0,12.5,4.5 \mathrm{~Hz}, 1 \mathrm{H}), 2.38(\mathrm{~d}, J=4.0 \mathrm{~Hz}, 1 \mathrm{H}), 2.61-2.76(\mathrm{~m}$, 2H), 3.48 (d, $J=8.0 \mathrm{~Hz}, 1 \mathrm{H}), 3.83$ (at, $J=7.5 \mathrm{~Hz}, 1 \mathrm{H}), 3.94$ (dd, $J=8.0,4.0 \mathrm{~Hz}, 1 \mathrm{H}), 4.44$ (dd, $J$ $=10.0,4.0 \mathrm{~Hz}, 1 \mathrm{H}), 4.57(\mathrm{~d}, J=12.0 \mathrm{~Hz}, 1 \mathrm{H}), 5.18(\mathrm{~d}, J=12.0 \mathrm{~Hz}, 1 \mathrm{H}), 7.20-7.32(\mathrm{~m}, 5 \mathrm{H}), 9.83$ $(\mathrm{t}, J=1.0 \mathrm{~Hz}, 1 \mathrm{H}) ;{ }^{13} \mathbf{C}$ NMR $\left(100 \mathrm{MHz}, \mathrm{CDCl}_{3}\right) \square 5.0,6.8,13.4,14.5,16.6,18.1,18.3,28.3$, 30.3, 39.8, 58.7, 72.2, 74.4, 74.6, 78.6, 85.5, 86.6, 126.3, 126.9, 127.9, 138.9, 201.2, 210.0; FTIR (thin film) $\square_{\max } 2958,2869,1766,1728,1464,1136,1090,1015,884,799,774,731,682$ $\mathrm{cm}^{-1}$; HRMS (ESI) calcd for $\mathrm{C}_{35} \mathrm{H}_{60} \mathrm{O}_{6} \mathrm{Si}_{2}[\mathrm{M}+\mathrm{Na}]^{+} 655.3826 \mathrm{~m} / \mathrm{z}$, found $655.3828 \mathrm{~m} / \mathrm{z}$.
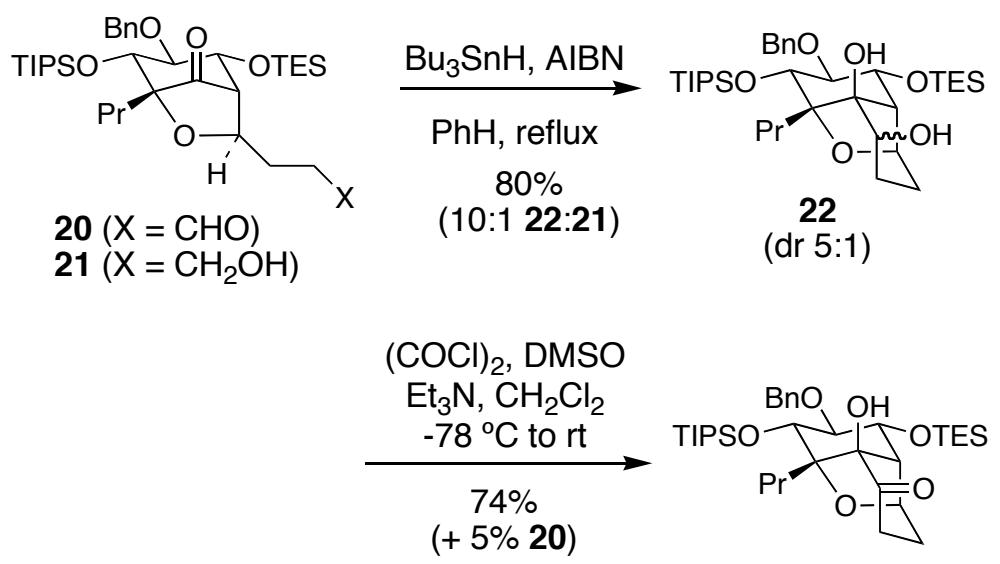

23

Ketone 23. To a solution of ketoaldehyde $20(33 \mathrm{mg}, 0.052 \mathrm{mmol})$ in $\mathrm{PhH}(2.6 \mathrm{~mL})$ was added $n-\mathrm{Bu}_{3} \mathrm{SnH}(0.021 \mathrm{~mL}, 0.078 \mathrm{mmol})$ and AIBN $(0.9 \mathrm{mg}, 0.005 \mathrm{mmol})$. The resulting mixture was heated at reflux for $16 \mathrm{~h}$, with additional portions of AIBN (ca. $1 \mathrm{mg}$ ) added after 3 $\mathrm{h}$ and $6 \mathrm{~h}$. The mixture was then cooled to room temperature and filtered through a short pad of silica gel. Concentration of the filtrate in vacuo and purification of the resultant residue by flash chromatography (5\% to $10 \%$ to $20 \%$ EtOAc in hexanes) gave diol 22 (26.5 $\mathrm{mg}, 0.042 \mathrm{mmol}$, 
$80 \%$; dr ca. 5:1), contaminated with ca. $10 \%$ of alcohol 21 . This mixture was used directly in the subsequent reaction without further purification.

To a solution of DMSO $(0.015 \mathrm{~mL}, 0.21 \mathrm{mmol})$ in $\mathrm{CH}_{2} \mathrm{Cl}_{2}(0.2 \mathrm{~mL})$ at $-78^{\circ} \mathrm{C}$ was added $(\mathrm{COCl})_{2}(0.009 \mathrm{~mL}, 0.11 \mathrm{mmol})$ dropwise via syringe. The mixture was stirred for $10 \mathrm{~min}$ at $-78{ }^{\circ} \mathrm{C}$, then a solution of diol 22 from above $(26.5 \mathrm{mg}, 0.042 \mathrm{mmol})$ in $\mathrm{CH}_{2} \mathrm{Cl}_{2}(0.4 \mathrm{~mL})$ was added via cannula. The resulting cloudy mixture was stirred at $-78{ }^{\circ} \mathrm{C}$ for $1 \mathrm{~h}$, and $\mathrm{Et}_{3} \mathrm{~N}(0.060$ $\mathrm{mL}, 0.42 \mathrm{mmol}$ ) was added. The mixture was then warmed to room temperature, extracted with $\mathrm{Et}_{2} \mathrm{O}$, and the organic extracts were dried $\left(\mathrm{MgSO}_{4}\right)$, filtered, and concentrated in vacuo. Purification of the residue via flash chromatography (3\% to 5\% to $10 \%$ EtOAc in hexanes) afforded ketone 23 (19.5 mg, $0.031 \mathrm{mmol}, 59 \%$ over two steps from 20) as a colorless oil, with

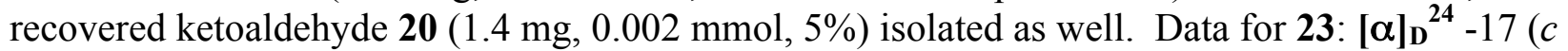
0.95, $\left.\mathrm{CHCl}_{3}\right) ;{ }^{1} \mathbf{H}$ NMR $\left(500 \mathrm{MHz}, \mathrm{CDCl}_{3}\right) \square 0.52-0.58(\mathrm{~m}, 6 \mathrm{H}), 0.79(\mathrm{t}, J=7.0 \mathrm{~Hz}, 3 \mathrm{H}), 0.90(\mathrm{t}$, $J=8.0 \mathrm{~Hz}, 9 \mathrm{H}), 0.95-1.04(\mathrm{~m}, 21 \mathrm{H}), 1.18-1.30(\mathrm{~m}, 1 \mathrm{H}), 1.65(\mathrm{ddd}, J=13.5,9.5,8.5 \mathrm{~Hz}, 1 \mathrm{H})$, $1.90(\mathrm{~d}, J=3.5 \mathrm{~Hz}, 1 \mathrm{H}), 1.96-2.08(\mathrm{~m}, 1 \mathrm{H}), 2.35$ (ddd, $J=13.5,8.5,4.0 \mathrm{~Hz}, 1 \mathrm{H}), 2.66-2.82(\mathrm{~m}$, 2H), $3.74(\mathrm{t}, J=8.0 \mathrm{~Hz}, 1 \mathrm{H}), 3.85(\mathrm{~s}, \mathrm{OH}), 4.14(\mathrm{~d}, J=8.0 \mathrm{~Hz}, 1 \mathrm{H}), 4.33(\mathrm{dd}, J=8.0,3.5 \mathrm{~Hz}$, $1 \mathrm{H}), 4.52(\mathrm{~d}, J=12.0 \mathrm{~Hz}, 1 \mathrm{H}), 4.63(\mathrm{~d}, J=4.0 \mathrm{~Hz}, 1 \mathrm{H}), 5.29(\mathrm{~d}, J=11.5 \mathrm{~Hz}, 1 \mathrm{H}), 7.20-7.27(\mathrm{~m}$, 1H), 7.29-7.36 (m, 4H); ${ }^{13} \mathbf{C}$ NMR (125 MHz, $\left.\mathrm{CDCl}_{3}\right) \square 5.2,6.8,13.8,14.6,16.8,18.4,18.7$, 29.7, 30.9, 33.0, 34.1, 54.7, 72.8, 73.1, 73.9, 74.6, 83.8, 85.0, 86.1, 126.7, 126.8, 127.9, 139.4, 209.5; FTIR (thin film) $\square_{\max } 3458,2958,2867,1708,1466,1388,1242,1116,1088,1014,948$, $884 \mathrm{~cm}^{-1}$; HRMS (ESI) calcd for $\mathrm{C}_{35} \mathrm{H}_{60} \mathrm{O}_{6} \mathrm{Si}_{2}[\mathrm{M}+\mathrm{Na}]^{+} 655.3826 \mathrm{~m} / \mathrm{z}$, found $655.3837 \mathrm{~m} / z$.

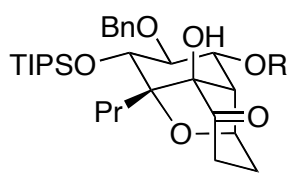

$23 \mathrm{R}=\mathrm{TES}$ $24 \mathrm{R}=\mathrm{H}$

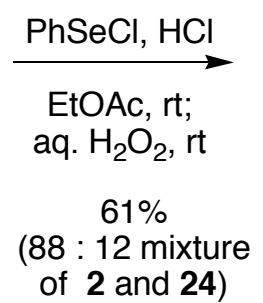

of 2 and 24)

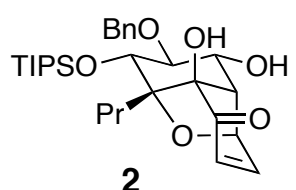

$41 \%$, ca. $95 \%$ pure after HPLC

Enone 2. To a solution of ketone $23(14 \mathrm{mg}, 0.022 \mathrm{mmol})$ in EtOAc $(0.23 \mathrm{~mL})$ at room temperature was added $\mathrm{PhSeCl}(5.1 \mathrm{mg}, 0.027 \mathrm{mmol})$ followed by conc. $\mathrm{HCl}(5 \square \mathrm{L})$. The resulting red-orange solution was stirred at room temperature for $6 \mathrm{~h}$, during which time the solution became yellow in color. The reaction was quenched with $\mathrm{H}_{2} \mathrm{O}$, extracted with $\mathrm{Et}_{2} \mathrm{O}$, and the organic layer was concentrated in vacuo to give the crude $\square$-selenoketone as an orange oil. This material was dissolved in $\mathrm{CH}_{2} \mathrm{Cl}_{2}$ (ca. $0.3 \mathrm{~mL}$ ), and pyridine (ca. $10 \square \mathrm{L}$ ) followed by $35 \%$ aq. $\mathrm{H}_{2} \mathrm{O}_{2}$ (ca. $30 \square \mathrm{L}$ ) were added. This mixture was stirred for $10 \mathrm{~min}$, during which time it turned from yellow to colorless; TLC analysis indicated the complete consumption of the selenide. The reaction was diluted with $\mathrm{Et}_{2} \mathrm{O}$, washed twice with water and once with brine, and the organic layer was dried $\left(\mathrm{MgSO}_{4}\right)$ and concentrated in vacuo. Purification of the resultant residue by flash chromatography ( $10 \%$ to $20 \%$ to $40 \%$ EtOAc in hexanes) provided an $88: 12$ mixture (as determined by ${ }^{1} \mathrm{H}$ NMR analysis) of enone 2 and ketone 24 (7 mg, $0.012 \mathrm{mmol}, 61 \%$ yield) as a colorless oil. This mixture was further purified by HPLC (55\% EtOAc in hexanes, 21 $\mathrm{mm}$ Dynamax 60A column, $\left.10 \mathrm{~mL} / \mathrm{min}, \mathrm{t}_{\mathrm{R}}=15.3 \mathrm{~min}\right)$ to provide enone $2(4.7 \mathrm{mg}, 0.091 \mathrm{mmol}$, $41 \%$ ) in ca. $95 \%$ purity (remainder 24) as a colorless oil. Data for 2: [ $]_{\mathbf{D}}{ }^{24}-60\left(\right.$ c $\left.0.47, \mathrm{CHCl}_{3}\right)$; 
${ }^{1} \mathbf{H}$ NMR $\left(500 \mathrm{MHz}, \mathrm{CDCl}_{3}\right) \square 0.78(\mathrm{t}, J=7.0 \mathrm{~Hz}, 3 \mathrm{H}), 1.04-1.16(\mathrm{~m}, 21 \mathrm{H}), 1.18-1.28(\mathrm{~m}, 3 \mathrm{H})$, $1.66(\mathrm{~d}, J=4.5 \mathrm{~Hz}, \mathrm{OH}), 1.80-1.88(\mathrm{~m}, 1 \mathrm{H}), 2.29(\mathrm{~d}, J=4.0 \mathrm{~Hz}, 1 \mathrm{H}), 3.78(\mathrm{t}, J=8.0 \mathrm{~Hz}, 1 \mathrm{H})$, 4.08 (s, OH), 4.13 (dt, $J=7.5,4.0 \mathrm{~Hz}, 1 \mathrm{H}), 4.34$ (d, $J=7.5 \mathrm{~Hz}, 1 \mathrm{H}), 4.74$ (dd, $J=6.0,1.5 \mathrm{~Hz}$, $1 \mathrm{H}), 4.81(\underline{\mathrm{AB}}, J=12.5 \mathrm{~Hz}, 1 \mathrm{H}), 4.87(\mathrm{AB}, J=12.5 \mathrm{~Hz}, 1 \mathrm{H}), 6.30(\mathrm{dd}, J=9.0,1.5 \mathrm{~Hz}, 1 \mathrm{H})$, 7.26-7.30 (m, 1H), 7.33-7.40 (m, 4H), 7.61 (dd, $J=8.5,5.5 \mathrm{~Hz}, 1 \mathrm{H}) ;{ }^{13} \mathbf{C}$ NMR $(100 \mathrm{MHz}$, $\left.\mathrm{CDCl}_{3}\right) \square 13.7,14.5,17.0,18.4,18.6,29.7,33.7,59.7,70.7,71.0,73.5,75.4,82.7,86.2,87.3$, 126.9, 127.4, 128.4, 128.8, 139.6, 156.6, 200.1; FTIR (thin film) $\square_{\max } 3455$ (br), 3064, 3031, 2963, 2944, 2867, 1681, 1466, 1374, 1249, 1226, 1139, 1114, 1086, 1049, 1016, 952, 884, 816 $\mathrm{cm}^{-1}$; HRMS (ESI) calcd for $\mathrm{C}_{29} \mathrm{H}_{44} \mathrm{O}_{6} \mathrm{Si}[\mathrm{M}+\mathrm{Na}]^{+} 539.2805 \mathrm{~m} / \mathrm{z}$, found $539.2814 \mathrm{~m} / \mathrm{z}(1.7$ $\mathrm{ppm})$. 